\title{
Disrupted circadian core-clock oscillations in Type 2 Diabetes are linked to altered rhythmic mitochondrial metabolism
}

Brendan M. Gabriel ${ }^{1,2 *}$, Ali Altıntaş ${ }^{3 *}$, Jonathon A.B. Smith ${ }^{1}$, Laura Sardon-Puig ${ }^{4}$, Xiping Zhang $^{5}$, Astrid L. Basse ${ }^{3}$, Rhianna C. Laker ${ }^{3}$, Hui Gao ${ }^{6}$, Zhengye Liu ${ }^{12}$, Lucile Dollet ${ }^{1}$, Jonas T. Treebak ${ }^{3}$, Antonio Zorzano ${ }^{7,8,9}$, Zhiguang Huo ${ }^{10}$, Mikael Rydén ${ }^{11}$, Johanna T. Lanner ${ }^{12}$, Karyn A. Esser ${ }^{5}$, Romain Barrès ${ }^{3}$, Nicolas J. Pillon ${ }^{1}$, Anna Krook ${ }^{1}$, Juleen R. Zierath ${ }^{1,3,4} \dagger$

${ }^{1}$ Department of Physiology and Pharmacology, Integrative Physiology, Karolinska Institutet, Stockholm, Sweden

${ }^{2}$ Aberdeen Cardiovascular \& Diabetes Centre, The Rowett Institute, University of Aberdeen, Aberdeen, UK.

${ }^{3}$ Novo Nordisk Foundation Center for Basic Metabolic Research, Faculty of Health and Medical Sciences, University of Copenhagen, Copenhagen, Denmark

${ }^{4}$ Department of Molecular Medicine and Surgery, Integrative Physiology, Karolinska Institutet, Stockholm, Sweden

${ }^{5}$ Department of Physiology and Functional Genomics, College of Medicine, University of Florida, Florida, USA

${ }^{6}$ Department of Biosciences and Nutrition (BioNut), Karolinska Institutet, Stockholm, Sweden

${ }^{7}$ Institute for Research in Biomedicine (IRB Barcelona). The Barcelona Institute of Science and Technology, Barcelona, Spain

${ }^{8}$ Departament de Bioquímica i Biomedicina Molecular, Facultat de Biologia, Barcelona, Spain

${ }^{9}$ CIBER de Diabetes y Enfermedades Metabólicas Asociadas (CIBERDEM), Instituto de Salud Carlos III, Spain

${ }^{10}$ Department of Biostatistics, University of Florida, Florida, USA

${ }^{11}$ Department of Medicine (H7), Unit for Endocrinology and Diabetes, Karolinska Institutet, Stockholm, Sweden

${ }^{12}$ Department of Physiology and Pharmacology, Molecular muscle physiology \& pathophysiology, Karolinska Institutet, Stockholm, Sweden

*Equal contribution

$\dagger$ Lead author:

Juleen R. Zierath, Integrative Physiology, Biomedicum, 17177 Stockholm, Sweden. Phone: +46 (0) 8-524 875 80. E-mail: Juleen.Zierath@ki.se 


\section{Summary}

Circadian rhythms are generated by an auto-regulatory feedback loop composed of transcriptional activators and repressors. Disruption of circadian rhythms contributes to Type 2 diabetes (T2D) pathogenesis. We elucidated whether altered circadian rhythmicity of clock genes is associated with metabolic dysfunction in T2D. Transcriptional cycling of core clock genes $A R N T L, C L O C K, C R Y 1$ and NRID1 was altered in skeletal muscle from individuals with T2D and this was coupled with reduced number and amplitude of cycling genes and disturbed circadian oxygen consumption. Mitochondrial associated genes were enriched for differential circadian amplitudes in T2D, and positively correlated with insulin sensitivity. ChIPsequencing identified CLOCK and BMAL1 binding to circadian mitochondrial genes associated with insulin sensitivity, implicating regulation by the core clock. Mitochondria disruption altered core-clock gene expression and free-radical production, phenomena that were restored by resveratrol treatment. We identify bi-directional communication between mitochondrial function and rhythmic gene expression, processes which are disturbed in diabetes. 


\section{Introduction}

Type 2 diabetes (T2D) is a growing global health problem, with skeletal muscle insulin resistance a primary defect in the pathology of this disease. While the etiology of this disease is complex, perturbed circadian rhythms from shift-work, sleep disorders, and social jet-lag are associated with obesity, T2D, and related co-morbidities (Mokhlesi et al., 2019; Shan et al., 2018; Tan et al., 2018; Vetter et al., 2018), highlighting the critical role of this circuit on metabolic health. Cell autonomous circadian rhythms are generated by a transcriptiontranslation auto-regulatory feedback loop composed of transcriptional activators CLOCK and BMAL1 $(A R N T L)$ and their target genes Period $(P E R)$, Cryptochrome $(C R Y)$, and REV-ERB $\alpha$ (NR1D1), which rhythmically accumulate and form a repressor complex that interacts with CLOCK and BMAL1 to inhibit transcription (Takahashi, 2015). Disruption of the molecularclock in skeletal muscle leads to obesity and insulin resistance in mouse models (Dyar et al., 2014; Harfmann et al., 2016; Schiaffino et al., 2016). While disrupted circadian rhythms alter metabolism, the extent to which these processes are impaired in people with T2D is unknown.

Several lines of evidence suggest that the link between dysregulated molecular-clock activity and T2D or insulin resistance may be tissue-dependent. In white adipose tissue, coreclock (PER1, PER2, PER3, CRY2, BMAL1, and DBP), clock-related (REVERB $)$ and metabolic $(P G C 1 \alpha)$ genes showed no difference of rhythm and amplitude between individuals with normal weight, obesity, or T2D over a time-course experiment (Otway et al., 2011). Conversely, in human leucocytes collected over a time-course experiment, mRNA expression of BMAL1, PER1, PER2 and PER3 was lower in people with T2D as compared to non-diabetic individuals (Ando et al., 2009). Additionally, BMAL1, PER1 and PER3 mRNA expression in leucocytes collected from people with $\mathrm{T} 2 \mathrm{D}$ is inversely correlated with $\mathrm{HbA}_{1 c}$ levels, suggesting an association of molecular-clock gene expression with T2D and insulin resistance. Furthermore, in pancreatic islets from individuals with T2D or healthy controls, PER2, PER3 
and CRY2 mRNA expression is positively correlated with islet insulin content and plasma HbAic levels (Stamenkovic et al., 2012). Thus, there may be tissue-specificity of molecularclock regulation, which contributes to clinical outcomes related to insulin sensitivity and T2D etiology. The underlying mechanisms regulating metabolic rhythmicity and, particularly, whether rhythmicity is lost in T2D remain incompletely understood.

At the cellular level, primary human myotubes maintain a circadian rhythm, with the amplitude of circadian gene $-R E V-E R B \alpha$ correlating with the metabolic disease state of the donor groups (Hansen et al., 2016). This apparent link between the skeletal muscle molecularclock and insulin sensitivity may be partly mediated by molecular-clock regulation of metabolic targets. ChIP-sequencing has revealed distinct skeletal muscle-specific BMAL1 and REV-ERB $\alpha$ cistromes (Dyar et al., 2018), with prominent molecular-clock targeted pathways including mitochondrial function, and glucose/lipid/protein metabolism (Dyar et al., 2018; Pastore and Hood, 2013). Moreover, these metabolic pathways may participate in retrograde signaling to control aspects of the molecular-clock. Pharmacological inhibition of DRP1, a key regulator of mitochondrial fission and metabolism, alters the period length of BMAL1 transcriptional activity in human fibroblasts (Schmitt et al., 2018). However, the signals and the clock-derived alterations that govern the rhythmicity of metabolism remain incompletely understood. Despite the growing evidence that several metabolic pathways are under circadian control, it is not clear whether circadian rhythmicity of the intrinsic molecular-clock is altered in T2D. Here we determined whether circadian control of gene expression and metabolism is altered at the cellular level in skeletal muscle from individuals with T2D. 


\section{Results}

\section{Intrinsically dysregulated circadian rhythm of gene expression in T2D myotubes}

Skeletal muscle biopsies were obtained from men with either T2D or normal glucose tolerance (NGT) (Figure 1A). Primary myotubes cultures were prepared, synchronized and harvested every 6 hours over 42 hours and the transcriptome was analyzed by RNA-sequencing (RNA-Seq). To determine whether genes displayed a rhythmic cycle over $\sim 24$ hours, we analyzed the RNA-seq expression values using "rhythmicity analysis incorporating nonparametric methods" (RAIN) algorithm with longitudinal method (Thaben and Westermark, 2014) (Supplemental Table 1). Myotubes from T2D donors displayed fewer cycling rhythmic genes as compared to myotubes from NGT donors and the rhythmic genes in NGT were significantly associated with the rhythmic genes in T2D (Figure 1B, Fisher exact test, $p=3.91 \mathrm{e}-$ 58). To induce acute insulin resistance and partly mimic a T2D milieu (Vlavcheski et al., 2020), myotubes from NGT and T2D donors were treated with high glucose and insulin (50 nM insulin, $25 \mathrm{mM}$ glucose) for 24 hours prior to serum shock. High concentration glucose and insulin treatment reduced the number of cycling genes in myotubes from both T2D and NGT donors (Figure 1C). We performed gene set enrichment analysis (GSEA) and found that reactome pathways (Jassal et al., 2020) (Figure 1D) that were enriched for cycling genes in the myotubes from the NGT donors included metabolic pathways such as 'Regulation of lipid metabolism by PPARA' and 'PPARA activates gene expression', while the reactome pathway 'Circadian Clock' was enriched in myotubes from the T2D donors. No reactome pathways were enriched in NGT (high glucose and insulin concentration), while 'Circadian Clock' was enriched in T2D (high glucose and insulin concentration). These data indicate that there are more metabolic genes cycling in cells from NGT donors. To assess the altered rhythmicity, so called differential rhythmicity, between NGT and T2D, the RNA-seq expression values from the NGT and T2D donors were compared to each other using DODR algorithm (Thaben and 
Westermark, 2014). BMAL1 (a.k.a. ARNTL)was the top differentially rhythmic gene (3 total, Supplemental Table 2) between NGT and T2D donors (Figure 1E, FDRDODR=0.096). Several molecular-clock genes, as well as clock-output genes, were exclusively cycling in either NGT or T2D, including $C L O C K, C R Y 1$ and, NR1D1 (FDRRAIN $<0.1$ ) (Figure 1E). These results indicate that myotubes from the NGT donors had more genes displaying rhythmic behavior. Moreover, cycling genes in NGT cells were enriched in metabolic pathways as compared to T2D cells, as well as cells treated with a high concentration of glucose and insulin. Additionally, several core clock genes displayed differential rhythmicity (DODR) or were exclusively rhythmic (RAIN) in myotubes from NGT and T2D donors.

\section{Altered peak-time signature of cycling genes in myotubes from T2D donors}

Analyzing the same RNA-sequencing data described in Figure 1, we used the RAIN algorithm to determine peak time of cycling genes in the myotubes. T2D displayed an altered pattern of cycling gene peaks as compared to NGT (Figure 2A and 2B). In percentage terms, T2D had the highest number of cycling genes displaying peaks at 24 hours, whereas NGT had the highest number of cycling genes displaying peaks at 12 hours. The number of cycling genes at each phase peak was more similar between T2D and NGT when cells were treated with a high concentration of glucose and insulin (Figure 2C and 2D). However, the peak at which both NGT and T2D had the largest amount of cycling genes remained consistent within diagnosis groups, with the highest number of cycling genes displaying peaks at 12 hours (NGT) and 24 hours (T2D), respectively. When considering the molecular-clock genes and clockoutput genes (Figure 2E), NPAS2, DBP and NR1D1 displayed different peak times between NGT and T2D. Additionally, NR1D1 and PER3 displayed different peak times between myotubes from NGT and NGT donors treated with a high concentration of glucose and insulin. We then performed a gene enrichment analysis (ORA) for each condition and gene peak time. Several metabolic pathways displayed enrichment for cycling genes at peak times 6 and 12 
hours in NGT cells, but not in any other condition (Figure 2F). For example, the pathway 'Metabolism of carbohydrates' was enriched at 6 hours only in NGT, whereas 'Regulation of lipid metabolism by PPARA' was enriched at 12 hours, in NGT alone. Thus, the myotubes from the NGT and T2D donors have an altered peak-time gene expression signature that is somewhat conserved even when cells are treated with a high concentration of glucose and insulin.

\section{Reduced amplitudes of rhythmic mitochondrial, and overall gene expression and ablated rhythmic mitochondrial metabolism in T2D}

An important factor which is often physiologically relevant in circadian and diurnal biology is the magnitude of cycling peaks and nadirs over the course of a cycle, which can be quantified by measuring the amplitude of cycling patterns. We used harmonic regression to determine relative amplitude of cycling gene expression in myotubes from NGT and T2D donors within the RNA-sequencing data-set. The mean $\log 2$ amplitude of cycling genes was lower in $\mathrm{T} 2 \mathrm{D}$ as compared to NGT (Figure 3A; $\mathrm{p}=2.2 \mathrm{e}-16$, two-sided Kolmogorov-Smirnov test). To determine if there was a specific cellular location that was enriched for amplitude divergence, we next performed GSEA using ranked log2-fold-changes of relative amplitudes. This analysis determined that the enriched Gene Ontology (GO) cellular components for reduced amplitudes in T2D compared to NGT were'Ficolin-1-rich granule lumen', 'Clathrin vesicle coat', 'Organelle inner membrane', and 'Mitochondrial inner membrane', 'Organelle Envelope' and ‘Mitochondrial Membrane’ (Figure 3B).

Given that reduced amplitude of a mitochondrial GO cellular component was observed in myotubes from the $\mathrm{T} 2 \mathrm{D}$ donors, and that $\mathrm{T} 2 \mathrm{D}$ is associated with reduced skeletal muscle mitochondrial function and metabolic inflexibility (Hesselink et al., 2016), we investigated whether synchronized myotubes from NGT and T2D donors displayed circadian oscillations of oxygen consumption rate (OCR; a proxy for mitochondrial metabolism, Figure 3C). We 
observed differential rhythmicity of basal OCR between NGT and T2D (DODR, FDR=4.08e09) (Figure 3D). Additionally, NGT displayed cycling OCR with a 16-hour period $($ FDRRAIN $=0.015)$ whereas T2D did not (nor any other period). Additionally, we performed a separate MitoStress test at a single timepoint (Zeitgeber Time: ZT24) after serum shock (Figure $\mathrm{S} 1)$. In this assay, there were no differences in mitochondrial function between the myotubes from NGT and T2D donors. Thus, the difference of rhythmic mitochondrial function between the myotubes from the NGT and T2D donors may be derived from temporal regulation of basal oxygen consumption, rather than differences in mitochondrial function per se. Overall, the data in Figure 3 demonstrate that myotubes from T2D donors have reduced mitochondrial metabolic rhythm in terms of both oxygen consumption rate and mitochondrial related gene amplitudes.

\section{The mitochondrial inner-membrane is enriched for lower circadian amplitudes and expression of genes that correlate with whole-body insulin sensitivity in T2D}

To test the in vivo clinical relevance of our findings in the primary myotube cultures, we performed a transcriptomic analysis on vastus lateralis muscle biopsies obtained from men with NGT $(n=22)$ or T2D $(n=22)$ at a single timepoint (Figure 4A). Additionally, a hyperinsulinemic-euglycemic clamp was performed to determine whole-body insulin sensitivity (M-value, analysis pipeline shown in Figure 4A). As expected, insulin sensitivity was greater in men with NGT as compared to T2D (Figure 4B, $<<0.0001$ ). The relationship between basal skeletal muscle gene expression and insulin sensitivity across the whole cohort was assessed by Spearman's rank correlation (Figure 4A). A gene-set enrichment analysis (GSEA) with ontology terms from GO cellular components was performed using Spearman's correlation coefficient as ranking metric and found that most of the enriched GO cellular components were related to the mitochondria (FDR $<0.10$, Figure $4 \mathrm{C}$ and $4 \mathrm{D}$ ). We then compared the enriched cellular components from the gene set enrichment analysis of log2-foldchange relative amplitudes that were down-regulated in T2D (Figure 4C). Two cellular 
components were consistent between these two analyses 'Mitochondrial inner membrane', and 'Organelle inner membrane' (Figure 4D). Our analysis points at the inner-mitochondria as a cellular location implicated in both the regulation of insulin sensitivity and associated with impaired cycling behaviors in skeletal myotubes.

\section{Mitochondrial pathways are enriched for circadian genes with Clock and Bmal1 binding, and are associated with whole-body insulin sensitivity}

In the current study, we describe intrinsically dysregulated rhythm, peak time, and amplitudes of cycling genes in myotubes from T2D as compared to NGT donors. Reduced amplitudes of cycling gene expression in T2D were particularly associated with mitochondrial related genes, and this was coupled with functionally dysregulated mitochondrial rhythmic metabolism. However, the regulatory directionality of these phenomena (i.e. rhythmic dysfunction of the molecular-clock and mitochondrial metabolism) is unclear. Whether both BMAL1 and CLOCK partially regulate mitochondrial metabolism in skeletal muscle is also unclear. We found that these molecular-clock genes were rhythmically dysregulated in myotubes from T2D as compared to NGT donors. Therefore, we tested whether BMAL1 and CLOCK binding was associated with mitochondrial genes involved in insulin sensitivity and dysregulated mitochondrial metabolic rhythms, which would indicate that dysregulated BMAL1 and CLOCK lead to disrupted mitochondrial rhythms in skeletal muscle of people with T2D. We performed ChIP-sequencing of mouse skeletal muscle using BMAL1 and CLOCK antibodies and assessed peaks closest to a gene's transcription start site. Firstly, we assessed whether circadian genes identified in myotubes from NGT and T2D donors were associated with BMAL1 and CLOCK binding to their counterpart genes (Figure 5A). Out of all the myotube conditions, only NGT was associated with BMAL1 and CLOCK binding, while T2D cells treated with a high concentration of glucose and insulin were associated with CLOCK binding exclusively. We then performed integrative clustering analysis between the 
ChIP-sequencing data, skeletal muscle gene expression correlated with insulin sensitivity, and circadian genes identified within myotubes from NGT and T2D donors incubated in the absence or presence of a high concentration of glucose and insulin. (Figure 5B). To integrate all different experimental outcomes, the data was presented as a binary matrix while 1 shows the presence and 0 shows the absence of rhythmicity, correlation or ChIP-seq peaks. The integrated data was subjected to clustering analysis, which resulted in 10 distinct clusters (Figure 5C). Clusters associated with BMAL1 and CLOCK binding were also associated with circadian rhythm in NGT (clusters 6, 7, and 8) and T2D (clusters 1, 2, 3, and 4). Several clusters demonstrated an association between T2D control conditions and T2D (high concentration of glucose and insulin) circadian genes, while NGT (high concentration of glucose and insulin)circadian genes clustered with very few other categories.

Genes that correlated with insulin sensitivity formed a cluster with BMAL1 and CLOCK binding, and circadian genes within myotubes from T2D donors (including when treated with a high concentration of glucose and insulin)(Cluster 3, and 4). We then performed gene set enrichment analysis of GO cellular components (Figure 5D). Of note, clusters 3 and 4 were enriched for genes associated mainly with the inner-mitochondria. There appeared to be few clustering events between insulin sensitivity-associated genes, genes with BMAL1 and CLOCK binding, and genes that were circadian in NGT. Thus, mitochondrial rhythmic metabolism within NGT may be partly independent of genes with CLOCK or BMAL1 binding.

\section{Pharmacological, genetic, and siRNA-mediated disruption of inner-mitochondrial metabolism results in altered clock-gene expression}

Our data provide evidence that mitochondrial metabolic rhythms in skeletal muscle from individuals with NGT may be partly independent of direct core-clock control. Additionally, internal mitochondrial gene expression was associated with whole-body insulin sensitivity, 
inner-mitochondrial genes also had reduced amplitudes in myotubes from T2D donors. Targets involved in internal mitochondrial functionality can act as regulators of molecular-clock expression and function (Lassiter et al., 2018; Schmitt et al., 2018). Thus, we hypothesized that internal mitochondrial functionality may play a role in regulating metabolic and molecular clock rhythms. To test this in myotubes from NGT donors, we used different compounds that target mitochondria, namely Carbonyl cyanide 4-(trifluoromethoxy)phenylhydrazone (FCCP), Oligomycin, and a mixture of Rotenone and Antimycin A (Rot/AA). After serum shock we incubated myotubes with these compounds for $4 \mathrm{~h}$ (ZT14-ZT18). We measured mRNA expression of molecular clock-associated genes $D B P$ (Figure 6A) and NR1D1 (Figure 6B), and found that $0.33 \mu \mathrm{M}$ of Rot/AA, and $2 \mu \mathrm{M}$ of FCCP increased mRNA expression of $D B P$ as compared to vehicle control-treated myotubes. Additionally, $2 \mu \mathrm{M}$ of FCCP, and $1 \mu \mathrm{M}$ of oligomycin increased mRNA expression of NR1D1 as compared to vehicle control-treated myotubes. These data provide evidence that manipulation of internal mitochondrial function may play a role in altering clock-associated gene expression in primary human myotubes. However, compounds such as these may have off-target effects, and thus we tested this hypothesis in other models.

We sought to manipulate a target that both regulated inner-mitochondrial function in myotubes and displayed diurnal behavior in vivo in skeletal muscle biopsies from individuals with NGT. Meeting these criteria, Optic Atrophy Protein 1 (OPA1) is an established regulator of inner-mitochondrial morphology and function in skeletal muscle (Lodi et al., 2011; Rodriguez-Nuevo et al., 2018; Sebastian et al., 2017; Tezze et al., 2017), and displays a daynight rhythm of protein abundance in skeletal muscle from healthy individuals, alongside oscillating mitochondrial function (van Moorsel et al., 2016). OPA1 did not display cycling mRNA in myotubes from NGT or T2D donors when adjusted for multiple comparisons. However, OMA1 and $H I G D 2 A$ are responsible for the cleavage and stabilization of OPA1 
protein (Anand et al., 2014; Salazar et al., 2019), respectively, and both of these genes displayed cycling mRNA patterns in myotube cultures from NGT, but not T2D donors (as determined via RAIN, Supplementary Figure 3, and Supplementary Table 1), suggesting circadian regulation of OPA1 protein. OPA1 basal gene expression also correlated with insulin sensitivity in the microarray data (Spearman's correlation, FDR=.002). To further elucidate the effect of mitochondrial disruption in relation to the molecular-clock machinery, we performed investigations in a skeletal muscle-specific $\mathrm{Opal}^{-/-}$mouse model. Molecular-clock genes Clock, Bmal1, Nrld1 and Per3 (Figure 6C) were decreased in Opa1 $1^{-/}$mice, suggesting retrograde signaling from OPA1 to the molecular-clock.

However, the $\mathrm{Opal}^{-/-}$mice we studied have a severe myopathic phenotype (RodriguezNuevo et al., 2018), we thus aimed to recapitulate this finding in myotube cultures from NGT donors (Figure 6D-6H). In these synchronized primary human skeletal myotubes, siRNAtargeted reduction of $O P A 1$ (Figure 6D) resulted in unchanged BMAL1 (Figure 6E) expression, whileNPAS2 (a paralogue and substitute of Clock (DeBruyne et al., 2007)) (Figure 6F), PER2 (Figure 6G), and PER3 (Figure 6H) all had altered $m R N A$ expression in the depleted OPA1 condition. OPA1 silencing also perturbed expression of complex $\mathrm{V}$ of the electron-transport chain (Figure 6 I). Collectively, these data suggest that manipulating inner-mitochondrial metabolism results in altered mRNA expression of clock genes.

\section{siRNA depletion of OPA1 increases mitochondrial reactive oxygen species, and OPA1- mediated changes in clock-gene expression can be restored by resveratrol treatment}

Our data suggest that the inner-mitochondrion is a modulator of the molecular-clock genes NPAS2, PER2 and PER3 in human skeletal muscle (Figure 6), implicating retrograde signaling between OPA1 and the molecular-clock. This signaling pathway may also be relevant for insulin sensitivity, since myotubes from T2D donors have downregulated circadian amplitudes 
of mitochondrial genes, ablated rhythmic metabolism, and altered core-clock rhythm. There are at least two plausible candidate pathways through which inner-mitochondrial-mediated signaling to the core-clock may occur (Figure 7A). Firstly, $\mathrm{NAD}^{+}$is a cofactor reflective of mitochondrial metabolism and $\mathrm{NAD}^{+}$-dependent sirtuins are regulators of molecular-clock genes (Peek et al., 2013). Secondly, oxygen sensing plays a key role in molecular signaling from the core-clock to mitochondria and vice-versa in skeletal muscle, via Hypoxia inducible factor-1 (HIF1- $\alpha$ ) (Peek et al., 2017). Interestingly, HIF1- $\alpha$ mRNA displayed circadian rhythms in the myotube cultures from the NGT but not the T2D donors in our RNA-sequencing data (determined via RAIN, Figure 7B). HIF1- $\alpha$ expression is driven by hypoxia in skeletal muscle, and regulated by reactive oxygen species (ROS) (Hoppeler et al., 2003). Thus, we explored the circadian mRNA patterns within our RNA-sequencing data of myotubes from T2D and NGT donors to identify genes that localize to mitochondria, which are associated with ROS processing and $\mathrm{NAD}^{+} / \mathrm{NADH}$ metabolism. Genes that were circadian in either T2D or NGT are highlighted (Figure 7C, with several antioxidant enzymes showing circadian regulation in NGT but not T2D (GPX1, GPX3, GPX4). Additionally, several genes involved in $\mathrm{NAD}^{+} / \mathrm{NADH}$ were circadian in NGT but not T2D (NARPT, NAMPT, NADSYN1), while NNT was circadian in T2D but not NGT. Of note, SOD2 (FDR=0.0005), GPX1 (FDR=0.03), and NAMPT $(\mathrm{FDR}=0.007)$ correlated with insulin sensitivity in our microarray data (Spearman's correlation). Given the apparent rhythmic differences in these metabolic pathways, we next assessed the corresponding metabolites in response to internal mitochondrion dysregulation. Control cells from NGT donors had differences in $\mathrm{NAD}^{+}$concentration over time, while cultures treated with siRNA targeting OPA1 had an $\mathrm{NAD}^{+}$concentration that did not differ over time However, there was no difference in $\mathrm{NAD}^{+}$concentration between siOPA1 and SCR Figure 4D). We then assessed mitochondrial ROS concentration using live-cell microscopy and measured MitoSOX fluorescence, indicative of mitochondrial ROS. OPA1-depleted 
myotubes had increased MitoSOX fluorescence when perfused with Tyrode's solution, as compared to scramble control (Figure 7E). We also treated OPA1-depleted myotubes with Resveratrol. Resveratrol can act as an antioxidant, and reduce HIF1- $\alpha$ activity, in addition to increasing SIRT1 activity (Repossi et al., 2020). Resveratrol reduced mitochondrial ROS levels in OPA1-depleted myotubes and rescued the OPA1-mediated changes in NPAS2 expression (Figure 7F and $\mathrm{G}$ ). Our results provide evidence to suggest that OPA1 regulation of mitochondrial ROS levels constitutes a feedback loop permitting bi-directional control of circadian metabolism in skeletal muscle.

\section{Discussion}

T2D shares several molecular and pathophysiological phenotypes with ageing (Cartee et al., 2016), which is associated with disruption of molecular-clock genes, in addition to dysregulation of other rhythmic biological processes (Zwighaft et al., 2015). Moreover, defective mitochondrial function and content is implicated in skeletal muscle insulin resistance and T2D pathogenesis (Ruegsegger et al., 2018; Schrauwen et al., 2010; Sebastian et al., 2017). Our data provide mechanistic insight into this metabolic dysfunction and show that myotubes from individuals with T2D have an intrinsically disrupted circadian rhythm, at the global transcriptomic and molecular-clock specific level. Furthermore, an acute treatment of myotubes with a high concentration of glucose and insulin, partially mimicking a diabetic milieu, somewhat replicates the reduced number of rhythmic genes in T2D. However, clear differences between T2D and treatment with high glucose and insulin concentrations exist, including the percentage of genes at each peak time, which were more similar within disease states, rather than treatment conditions. These data suggest a longer-term, intrinsic conservation of the T2D circadian signature in primary myocytes. This finding would appear consistent with data demonstrating that induced pluripotent stem cells from donors with T2D that were differentiated into myoblasts had multiple defects as compared to healthy subjects, 
including reduced insulin-stimulated glucose uptake and reduced mitochondrial oxidation (Batista et al., 2020). These T2D-associated defects were conserved despite the robust manipulations that the cells undergo. How cultured myocytes from T2D donors preserve a dysfunctional phenotype, including insulin resistance (Bouzakri and Zierath, 2007; Sarabia et al., 1992), has not been fully elucidated, but likely reflects genetic background and epigenetic mechanisms. Our data reveal that an intrinsic disruption of circadian biology in skeletal muscle may predispose to insulin resistance and metabolic disease. The cause of this disruption is unknown, but in addition to inherited factors, cumulative effects of sedentary lifestyle, sleep deprivation, nutritional and hormonal factors are likely to play a role (Gabriel and Zierath, 2019; Zimmet et al., 2016).

Our results demonstrate that dysregulated rhythmic mitochondrial metabolism may play a role in mediating the disrupted rhythmic cellular metabolism and molecular-clock machinery in primary myocytes from individuals with T2D. Mitochondrial diurnal rhythms in oxidative capacity and oxygen metabolism have been demonstrated in several tissues, including skeletal muscle in vivo (Hansen et al., 2016). These skeletal muscle diurnal mitochondrial rhythms are more likely to emanate from mitochondrial membrane dynamic processes, regulated by rhythmic proteins such as OPA1 and FIS1, rather than a day-night rhythm of mitochondrial biogenesis and content (de Goede et al., 2018; van Moorsel et al., 2016). Mitochondrial membrane dynamics demonstrate circadian behavior in several peripheral tissues (de Goede et al., 2018), however, transcriptional control of these pathways is tissue-specific (Schrauwen et al., 2010; Weaver et al., 2014). For example, while Drp-1, Mfn1/2, and Opal are not direct targets of the clock machinery in liver (Jacobi et al., 2015), Mfnl and Opal are decreased in cardiac muscle of Bmall $^{-/}$mice (Kohsaka et al., 2014).

In the current study, ChIP-sequencing revealed that the inner-mitochondrial genes highly associated with insulin sensitivity also were direct targets of both CLOCK and BMAL1 in 
skeletal muscle. Moreover, some of these genes were also circadian in myotube cultures from donors with T2D. However, genes with CLOCK and BMAL1 binding did not coalesce with inner-mitochondrial genes in myotubes from donors with NGT, despite the similar enrichment of these pathways when assessing genes with reduced rhythmic amplitudes in T2D and genes that correlated with insulin sensitivity in vivo. Mitochondrial metabolism plays a role in bidirectional regulation of diurnal metabolism in peripheral tissues (Manella and Asher, 2016). Here we report that myotube cultures from donors with NGT displayed more robust mitochondrial metabolic rhythms as compared with T2D. Mitochondrial rhythms in individuals with NGT may help to maintain the diurnal metabolic rhythm in skeletal muscle. Additionally, manipulating mitochondrial membrane metabolism may alter the rhythmic expression of molecular-clock genes (Lassiter et al., 2018; Schmitt et al., 2018), although the signaling mechanism between mitochondrial metabolism and the molecular-clock has not been elucidated. Our results provide evidence that $O P A 1$ depletion disrupts molecular-clock genes NPAS2, PER2 and PER3 in primary myocytes.

Mitochondrial respiration and ROS production are tightly linked, and alongside mitochondrial capacity and oxygen consumption, antioxidant proteins and ROS production also display circadian activity (de Goede et al., 2018). In our study, mRNA expression of antioxidant enzymes that localize to mitochondria ( $G P X 1, G P X 3, G P X 4)$ were rhythmic in myotube cultures from donors with NGT, but not T2D. The aforementioned enzymes are all involved in the detoxification of hydrogen peroxide, a known regulator of HIF1- $\alpha$ activity (Hoppeler et al., 2003), which was also rhythmic in myotube cultures from donors with NGT, but not T2D. HIF1- $\alpha$ plays a key role in mediating signaling from the core-clock to mitochondria and vice-versa in skeletal muscle. Our data indicate that altered rhythms of mitochondrial respiration and ROS handling in myotube cultures from donors with T2D may be partly responsible for the ablated rhythm of HIF1- $\alpha$ expression. In support of this, the OPA1- 
mediated increase in NPAS2 expression and mitochondrial ROS were rescued by treatment with resveratrol, an antioxidant and HIF $1-\alpha$ modulator. Collectively, our data show cross-talk between the inner-mitochondrion and the molecular-clock, with genes of these pathways rhythmically dysregulated in T2D. Dysfunction of the inner-mitochondrion in peripheral tissues is implicated in the pathogenesis of insulin resistance and T2D, concomitant with increased ROS production, decreased oxidative capacity, and reduced metabolic flexibility (Schrauwen et al., 2010). This dysfunction may also play a role in regulating intrinsically altered metabolic, and molecular-clock rhythms in T2D.

In conclusion, we show disturbances in the intrinsic rhythmicity of gene expression and metabolism in skeletal muscle cells of individuals with T2D. This dysregulation is evident in myocytes in the absence of systemic factors, hormones, nutritional cues, or direct influence of the central or other peripheral clocks. Moreover, circadian inner-mitochondrion gene amplitudes are downregulated in T2D. These genes are also associated with insulin sensitivity and are under bi-directional regulation of circadian metabolism in skeletal muscle. The dysregulation of circadian metabolism in skeletal muscle of people with T2D underscores the need to take circadian biology into account and consider approaches in chrono-medicine (Roenneberg and Merrow, 2016) when prescribing pharmacological therapy, particularly treatments that affect mitochondrial function (Fontaine, 2018). Our findings provide mechanistic insight into T2D pathophysiology and have clinical implications into the link between insulin sensitivity and environmental triggers that are associated with altered metabolism, including impaired sleeping-patterns, social jet-lag, or shift-work. 


\section{Acknowledgments}

The authors are supported by grants from the AstraZeneca SciLifeLab Research Programme, Novo Nordisk Foundation (NNF14OC0011493, NNF14OC0009941, NNF17OC0030088), Swedish Diabetes Foundation (DIA2018-357), Swedish Research Council (2015-00165, 201802389), the Strategic Research Programme in Diabetes at Karolinska Institutet (2009-1068), the Stockholm County Council (SLL20170159) and the Swedish Research Council for Sport Science (P2019-0140). Brendan M. Gabriel was supported by fellowships from the Novo Nordisk Foundation (NNF19OC0055072), the Wenner-Gren Foundation, and an Albert Renold Travel Fellowship from the European Foundation for the Study of Diabetes and an Eric Reid Fund for Methodology from the Biochemical Society. Nicolas J. Pillon and Laura Sardon Puig were supported by an Individual Fellowship from the Marie Skłodowska-Curie Actions (European Commission: 704978, 675610). Xiping Zhang and Karyn Esser were supported by NIH R01AR066082. Nicolas J. Pillon were supported by grants from the Sigurd och Elsa Goljes Minne and Lars Hierta Memorial Foundations (Sweden). We acknowledge the Beta Cell in-vivo Imaging/ Extracellular Flux Analysis core facility supported by the Strategic Research Program in Diabetes for the usage of the Seahorse flux analyzer. Additional support was received from the Novo Nordisk Foundation Center for Basic Metabolic Research at the University of Copenhagen (NNF18CC0034900). The Novo Nordisk Foundation Center for Basic Metabolic Research is an independent research center at the University of Copenhagen, partially funded by an unrestricted donation from the Novo Nordisk Foundation. We acknowledge the Single-Cell Omics platform at the Novo Nordisk Foundation Center for Basic Metabolic Research for technical and computational expertise and support. 


\section{Author Contributions}

Conceptualization: BMG, AA, MR, KAE, AK, JRZ

Methodology: BMG, AA, LSP, JABS, NJP, ZL, JTL AZ, KAE, RB, MR, AK, JRZ

Formal Analysis: BMG, AA

Investigation: BMG, AA, LSP, JABS, ZL, LD, AA, XZ, ALB, RCL, NJP

Resources: JTT, AZ, JTL, KAE, RB, MR, NJP, AK, JRZ

Writing - Original Draft: BMG, JRZ

Writing -Review \& Editing: BMG, AA, LSP, JABS, XZ, ALB, RCL, HG, ZL, LD, JTT, AZ, ZH, MR, JTL, KAE, RB, NJP, AK, JRZ

Funding Acquisition: BMG, NJP, JTT, JTL, AZ, KAE, RB, MR, AK, JRZ

\section{References}

Abu-Jamous, B., and Kelly, S. (2018). Clust: automatic extraction of optimal co-expressed gene clusters from gene expression data. Genome Biology 19, 172-172.

Anand, R., Wai, T., Baker, M.J., Kladt, N., Schauss, A.C., Rugarli, E., and Langer, T. (2014). The i-AAA protease YME1L and OMA1 cleave OPA1 to balance mitochondrial fusion and fission. J Cell Biol 204, 919-929.

Ando, H., Takamura, T., Matsuzawa-Nagata, N., Shima, K.R., Eto, T., Misu, H., Shiramoto, M., Tsuru, T., Irie, S., Fujimura, A., et al. (2009). Clock gene expression in peripheral leucocytes of patients with type 2 diabetes. Diabetologia 52, 329-335.

Batista, T.M., Jayavelu, A.K., Wewer Albrechtsen, N.J., Iovino, S., Lebastchi, J., Pan, H., Dreyfuss, J.M., Krook, A., Zierath, J.R., Mann, M., et al. (2020). A cell-autonomous signature of dysregulated protein phosphorylation underlies muscle insulin resistance in Type 2 Diabetes. Cell Metab 32, 844-859 e845.

Bouzakri, K., and Zierath, J.R. (2007). MAP4K4 gene silencing in human skeletal muscle prevents tumor necrosis factor-alpha-induced insulin resistance. J Biol Chem 282, 7783-7789.

Cartee, G.D., Hepple, R.T., Bamman, M.M., and Zierath, J.R. (2016). Exercise promotes healthy aging of skeletal muscle. Cell Metab 23, 1034-1047.

de Goede, P., Wefers, J., Brombacher, E.C., Schrauwen, P., and Kalsbeek, A. (2018). Circadian rhythms in mitochondrial respiration. J Mol Endocrinol 60, R115-R130.

DeBruyne, J.P., Weaver, D.R., and Reppert, S.M. (2007). CLOCK and NPAS2 have overlapping roles in the suprachiasmatic circadian clock. Nat Neurosci 10, 543-545.

Dyar, K.A., Ciciliot, S., Wright, L.E., Bienso, R.S., Tagliazucchi, G.M., Patel, V.R., Forcato, M., Paz, M.I., Gudiksen, A., Solagna, F., et al. (2014). Muscle insulin sensitivity and glucose metabolism are controlled by the intrinsic muscle clock. Mol Metab 3, 29-41.

Dyar, K.A., Hubert, M.J., Mir, A.A., Ciciliot, S., Lutter, D., Greulich, F., Quagliarini, F., Kleinert, M., Fischer, K., Eichmann, T.O., et al. (2018). Transcriptional programming of lipid and amino acid metabolism by the skeletal muscle circadian clock. PLoS Biol 16, e2005886.

Fontaine, E. (2018). Metformin-induced mitochondrial complex I inhibition: Facts, uncertainties, and consequences. Front Endocrinol (Lausanne) 9, 753. 
Gabriel, B.M., and Zierath, J.R. (2019). Circadian rhythms and exercise - re-setting the clock in metabolic disease. Nat Rev Endocrinol 15, 197-206.

Hansen, J., Timmers, S., Moonen-Kornips, E., Duez, H., Staels, B., Hesselink, M.K., and Schrauwen, P. (2016). Synchronized human skeletal myotubes of lean, obese and type 2 diabetic patients maintain circadian oscillation of clock genes. Sci Rep 6, 35047.

Harfmann, B.D., Schroder, E.A., Kachman, M.T., Hodge, B.A., Zhang, X., and Esser, K.A. (2016). Muscle-specific loss of Bmall leads to disrupted tissue glucose metabolism and systemic glucose homeostasis. Skelet Muscle 6, 12.

Hesselink, M.K., Schrauwen-Hinderling, V., and Schrauwen, P. (2016). Skeletal muscle mitochondria as a target to prevent or treat type 2 diabetes mellitus. Nat Rev Endocrinol 12, 633-645.

Hoppeler, H., Vogt, M., Weibel, E.R., and Fluck, M. (2003). Response of skeletal muscle mitochondria to hypoxia. Exp Physiol 88, 109-119.

Jacobi, D., Liu, S., Burkewitz, K., Kory, N., Knudsen, N.H., Alexander, R.K., Unluturk, U., Li, X., Kong, X., Hyde, A.L., et al. (2015). Hepatic Bmall regulates rhythmic mitochondrial dynamics and promotes metabolic fitness. Cell Metab 22, 709-720.

Jassal, B., Matthews, L., Viteri, G., Gong, C., Lorente, P., Fabregat, A., Sidiropoulos, K., Cook, J., Gillespie, M., Haw, R., et al. (2020). The reactome pathway knowledgebase. Nucleic Acids Res 48, D498-d503.

Kohsaka, A., Das, P., Hashimoto, I., Nakao, T., Deguchi, Y., Gouraud, S.S., Waki, H., Muragaki, Y., and Maeda, M. (2014). The circadian clock maintains cardiac function by regulating mitochondrial metabolism in mice. PLoS One 9, e112811.

Lassiter, D.G., Sjogren, R.J.O., Gabriel, B.M., Krook, A., and Zierath, J.R. (2018). AMPK activation negatively regulates GDAP1, which influences metabolic processes and circadian gene expression in skeletal muscle. Mol Metab 16, 12-23.

Lodi, R., Tonon, C., Valentino, M.L., Manners, D., Testa, C., Malucelli, E., La Morgia, C., Barboni, P., Carbonelli, M., Schimpf, S., et al. (2011). Defective mitochondrial adenosine triphosphate production in skeletal muscle from patients with dominant optic atrophy due to OPA1 mutations. Arch Neurol 68, 67-73.

Manella, G., and Asher, G. (2016). The circadian nature of mitochondrial biology. Front Endocrinol (Lausanne) 7, 162.

Mokhlesi, B., Temple, K.A., Tjaden, A.H., Edelstein, S.L., Utzschneider, K.M., Nadeau, K.J., Hannon, T.S., Sam, S., Barengolts, E., Manchanda, S., et al. (2019). Association of self-reported sleep and circadian measures with glycemia in adults with prediabetes or recently diagnosed untreated type 2 diabetes. Diabetes Care 42, 1326.

Otway, D.T., Mantele, S., Bretschneider, S., Wright, J., Trayhurn, P., Skene, D.J., Robertson, M.D., and Johnston, J.D. (2011). Rhythmic diurnal gene expression in human adipose tissue from individuals who are lean, overweight, and type 2 diabetic. Diabetes 60 , $1577-1581$.

Pastore, S., and Hood, D.A. (2013). Endurance training ameliorates the metabolic and performance characteristics of circadian Clock mutant mice. J Appl Physiol (1985) 114, 1076-1084.

Peek, C.B., Affinati, A.H., Ramsey, K.M., Kuo, H.Y., Yu, W., Sena, L.A., Ilkayeva, O., Marcheva, B., Kobayashi, Y., Omura, C., et al. (2013). Circadian clock NAD+ cycle drives mitochondrial oxidative metabolism in mice. Science 342, 1243417.

Peek, C.B., Levine, D.C., Cedernaes, J., Taguchi, A., Kobayashi, Y., Tsai, S.J., Bonar, N.A., McNulty, M.R., Ramsey, K.M., and Bass, J. (2017). Circadian clock interaction with HIF1alpha mediates oxygenic metabolism and anaerobic glycolysis in skeletal muscle. Cell Metab 25, 86-92. 
Repossi, G., Das, U.N., and Eynard, A.R. (2020). Molecular basis of the beneficial actions of resveratrol. Arch Med Res 51, 105-114.

Rodriguez-Nuevo, A., Diaz-Ramos, A., Noguera, E., Diaz-Saez, F., Duran, X., Munoz, J.P., Romero, M., Plana, N., Sebastian, D., Tezze, C., et al. (2018). Mitochondrial DNA and TLR9 drive muscle inflammation upon Opa1 deficiency. EMBO J 37.

Roenneberg, T., and Merrow, M. (2016). The circadian clock and human health. Curr Biol 26, R432-443.

Ruegsegger, G.N., Creo, A.L., Cortes, T.M., Dasari, S., and Nair, K.S. (2018). Altered mitochondrial function in insulin-deficient and insulin-resistant states. J Clin Invest 128, 3671-3681.

Salazar, C., Elorza, A.A., Cofre, G., Ruiz-Hincapie, P., Shirihai, O., and Ruiz, L.M. (2019). The OXPHOS supercomplex assembly factor HIG2A responds to changes in energetic metabolism and cell cycle. J Cell Physiol 234, 17405-17419.

Sarabia, V., Lam, L., Burdett, E., Leiter, L.A., and Klip, A. (1992). Glucose transport in human skeletal muscle cells in culture. Stimulation by insulin and metformin. J Clin Invest 90, 1386-1395.

Schiaffino, S., Blaauw, B., and Dyar, K.A. (2016). The functional significance of the skeletal muscle clock: lessons from Bmal1 knockout models. Skelet Muscle 6, 33.

Schmitt, K., Grimm, A., Dallmann, R., Oettinghaus, B., Restelli, L.M., Witzig, M., Ishihara, N., Mihara, K., Ripperger, J.A., Albrecht, U., et al. (2018). Circadian control of DRP1 activity regulates mitochondrial dynamics and bioenergetics. Cell Metab 27, 657-666 e655.

Schrauwen, P., Schrauwen-Hinderling, V., Hoeks, J., and Hesselink, M.K. (2010). Mitochondrial dysfunction and lipotoxicity. Biochim Biophys Acta 1801, 266-271.

Sebastian, D., Palacin, M., and Zorzano, A. (2017). Mitochondrial Dynamics: Coupling Mitochondrial Fitness with Healthy Aging. Trends Mol Med 23, 201-215.

Shan, Z., Li, Y., Zong, G., Guo, Y., Li, J., Manson, J.E., Hu, F.B., Willett, W.C., Schernhammer, E.S., and Bhupathiraju, S.N. (2018). Rotating night shift work and adherence to unhealthy lifestyle in predicting risk of type 2 diabetes: results from two large US cohorts of female nurses. BMJ 363, k4641.

Stamenkovic, J.A., Olsson, A.H., Nagorny, C.L., Malmgren, S., Dekker-Nitert, M., Ling, C., and Mulder, H. (2012). Regulation of core clock genes in human islets. Metabolism 61, 978-985.

Takahashi, J.S. (2015). Molecular components of the circadian clock in mammals. Diabetes, Obesity and Metabolism 17, 6-11.

Tan, X., Chapman, C.D., Cedernaes, J., and Benedict, C. (2018). Association between long sleep duration and increased risk of obesity and type 2 diabetes: A review of possible mechanisms. Sleep Medicine Reviews 40, 127-134.

Tezze, C., Romanello, V., Desbats, M.A., Fadini, G.P., Albiero, M., Favaro, G., Ciciliot, S., Soriano, M.E., Morbidoni, V., Cerqua, C., et al. (2017). Age-associated loss of OPA1 in muscle impacts muscle mass, metabolic homeostasis, systemic inflammation, and epithelial senescence. Cell Metab 25, 1374-1389 e1376.

Thaben, P.F., and Westermark, P.O. (2014). Detecting rhythms in time series with RAIN. J Biol Rhythms 29, 391-400.

van Moorsel, D., Hansen, J., Havekes, B., Scheer, F., Jorgensen, J.A., Hoeks, J., SchrauwenHinderling, V.B., Duez, H., Lefebvre, P., Schaper, N.C., et al. (2016). Demonstration of a day-night rhythm in human skeletal muscle oxidative capacity. Mol Metab 5, 635-645. 
Vetter, C., Dashti, H.S., Lane, J.M., Anderson, S.G., Schernhammer, E.S., Rutter, M.K., Saxena, R., and Scheer, F. (2018). Night shift work, genetic risk, and type 2 diabetes in the UK Biobank. Diabetes Care.

Vlavcheski, F., Den Hartogh, D.J., Giacca, A., and Tsiani, E. (2020). Amelioration of highinsulin-induced skeletal muscle cell insulin resistance by resveratrol is linked to activation of AMPK and restoration of GLUT4 translocation. Nutrients 12, 914.

Weaver, D., Eisner, V., Liu, X., Varnai, P., Hunyady, L., Gross, A., and Hajnoczky, G. (2014). Distribution and apoptotic function of outer membrane proteins depend on mitochondrial fusion. Mol Cell 54, 870-878.

Zimmet, P., Alberti, K.G., Magliano, D.J., and Bennett, P.H. (2016). Diabetes mellitus statistics on prevalence and mortality: facts and fallacies. Nat Rev Endocrinol 12, 616-622.

Zwighaft, Z., Aviram, R., Shalev, M., Rousso-Noori, L., Kraut-Cohen, J., Golik, M., Brandis, A., Reinke, H., Aharoni, A., Kahana, C., et al. (2015). Circadian clock control by polyamine levels through a mechanism that declines with age. Cell Metab 22, 874885. 


\section{Figure legends}

Figure 1. Intrinsically dysregulated circadian rhythm of gene expression in T2D

(A) Schematic overview of primary cell culture circadian experiment and RNA-Sequencing. RNA-seq of primary human skeletal muscle cells from men with NGT $(n=7)$ or T2D $(n=5)$.

(B) Venn diagram showing the overlapping rhythmic genes between NGT and T2D. Overlapping rhythmic genes were significantly enriched (Fisher's exact test, $\mathrm{p}=3.91 \mathrm{e}-58$; Odds-ratio, $\mathrm{OR}=2.54$, background $=18,483$ )

(C) Number of circadian genes identified via RAIN analysis (FDR $<0.10)$.

(D) Circadian gene enrichment results using over-representation analysis (Fisher's exact test) and Reactome pathways. Circadian genes identified via RAIN analysis $(\mathrm{FDR}<0.10)$ and top 10 enriched Reactome pathways are shown $(\mathrm{FDR}<0.10)$.

(E) Circadian rhythmicity of core clock genes. Red=T2D, Black=NGT. Lines show the harmonic regression fits and solid line indicates circadian $(\mathrm{FDR}<0.10)$ genes while dashed lines indicate non-circadian genes. Time points are hours post-synchronization.

\section{Figure 2. Altered peak-time signature of cycling genes in T2D}

(A) Number of circadian genes at each peak time for control treatments.

(B) Percentage of circadian genes at each peak time for control treatments.

(C) Number of circadian genes at each peak time when treated with high concentrations of glucose and insulin.

(D) Percentage of circadian genes at each peak time when treated with high concentrations of glucose and insulin. (E) Heatmap showing the peak times of core clock genes for control conditions and cells treated with high concentrations of glucose and insulin. Colors represent the peak times. Hierarchical clustering was performed by using geodesic distance and 'ward.D2' algorithm. Star signs represent the adjusted p-values from rhythmicity analysis 
(RAIN): “***” $(\mathrm{p}<0.001), “ * * ”(0.001-0.01), “ * ”(0.01-0.05), “ . ”(0.05-0.1)$ and empty boxes $(0.1-1)$.

(F) Reactome pathways enriched at each time point in NGT (control), T2D (control), NGT (high concentration of glucose and insulin.), and T2D (high concentration of glucose and insulin.).

Figure 3. Reduced amplitudes of rhythmic mitochondrial, and overall gene expression and ablated rhythmic mitochondrial metabolism in T2D

(A) $\log 2$ relative amplitude of circadian genes for T2D as compared to NGT, relative amplitudes determined via harmonic regression with first degree polynomial trend using mean centered data. Histogram of circadian genes (red) and all genes (black), log2 relative amplitude of T2D vs NGT. The main panel shows count histogram of log2 relative amplitudes of circadian genes (red) and noncircadian genes (black). Panel on right top show the density distributions and ECDF (Empirical Cumulative Distribution Function). Red=circadian, determined via RAIN algorithm $(\mathrm{FDR}<0.1)$, Black=non-circadian. Log2 relative amplitude distributions were significantly different in circadian genes compared to noncircadian genes ( $p=1.28 \mathrm{e}-6$, two-sided Kolmogorov-Smirnov test). $\mu=$ population mean.

(B) GO term - Gene interaction plot of top enriched cellular component for ranked log2 relative amplitude changes of GO terms mitochondrion, mitochondrial membrane, organelle envelope.

(C) Schematic of circadian basal cellular oxygen consumption rate time-course experiment.

(D) Relative oxygen consumption rate of synchronized myotube cultures from donors with NGT (black) versus T2D (red), as measured by Seahorse XF Analyzer (Agilent) for $n=5$ individuals in both groups. Differential rhythmicity (DODR, period $=16$ hours) and rhythmicity (RAIN) analysis statistics are shown in table inset. See also Figure S1. 
Figure 4. Mitochondrial pathways are enriched for lower T2D circadian amplitudes and genes that correlate with whole-body insulin sensitivity

(A) Schematic of experimental design.

(B) Insulin sensitivity (M-value) of T2D and NGT participants. Student's t-test.

(C) Enrichment analysis based on microarray of skeletal muscle biopsies obtained in the fasted state from 22 men with NGT and 22 men with T2D obtained in the fasted state. Differentially enriched GO cellular components (GO:CC), ranked by FDR-values. Column 1 and 2: Top enriched cellular components for ranked $\log 2$ relative amplitude changes, Column 3: Pathway enrichment calculated from genes correlating FDR $<0.10$ ) with $\mathrm{M}$-value (whole-body insulin sensitivity) in vivo. There were no enriched pathways in the T2D versus NGT (high concentration of glucose and insulin.) (Up) condition.

(D) GO term - Gene interaction plot of GO:CC enrichments calculated from genes correlating $(\mathrm{FDR}<0.1) \mathrm{M}$-value (whole-body insulin sensitivity) in vivo: organelle inner membrane, mitochondrial inner membrane, mitochondrial protein complex, mitochondrial matrix, oxidoreductase complex.

Figure 5. Mitochondrial pathways are enriched for circadian genes with Clock and Bmal1 binding, and are associated with whole-body insulin sensitivity

(A) Enrichment analysis between circadian genes and ChIP-seq peaks. The association between circadian genes in each disease-treatment group and BMAL1/CLOCK bound genes were tested using Fisher's exact test and p-values are adjusted for multiple testing with Benjamini-Hochberg method. Columns: BMAL1/CLOCK ChIP-seq experiments; rows: Rhythmic genes in NGT and T2D for both control conditions and high concentration of glucose and insulin treatment groups; numbers: adjusted p-values (ns: not significant); colors: Jaccard similarity index indicating the percentage of genes overlapping in each dataset.

(B) Schematic of experimental design used for the integrative analysis. 
(C) Heatmap showing the clusters from the integrative analysis. Binary data used for clustering with "clust" algorithm (Abu-Jamous and Kelly, 2018) and resulted in 10 clusters. Columns "NGT (Control)", “T2D (Control)”, NGT (high concentration of glucose and insulin.)”, "T2D (high concentration of glucose and insulin.)" represents circadian (black) and noncircadian (white) genes; "M-value cor" shows whether a significant (black) correlation between the insulin sensitivity metric (M-value) and basal gene expression; "BMAL1" and "CLOCK" show whether a ChIP-seq peak for the given protein was detected (black) or not (white).

(D) Gene enrichment analysis using over-representation (Fisher's exact test) with GO cellular components was performed for each cluster identified in (B).

\section{Figure 6. Pharmacological, genetic, and siRNA-mediated disruption of inner-} mitochondrial metabolism results in altered clock-gene expression

(A) mRNA expression of molecular clock-associated gene DBP in myotubes from NGT donors ( $\mathrm{n}=4-6$ donors). One-way ANOVA, ${ }^{*}=\mathrm{p}<0.05$ compared to vehicle control (Con.).

(B) mRNA expression of molecular clock-associated gene NR1D1 in myotubes from NGT donors ( $\mathrm{n}=4-6$ donors). One-way ANOVA, $*=\mathrm{p}<0.05$ compared to vehicle control (Con.).

(C) OPA1 and Clock gene expression (Bmal1, Clock, Nr1d1, Per2, Per3) from Opa1 MKOOpal skeletal muscle Opal $^{-/-}$mice $(\mathrm{n}=8)$ relative to control $(\mathrm{n}=7)$. Black=siRNA-target Opal gene expression, purple=core-clock/associated gene expression One-way ANOVA, $* * *=p<0.001, *=p<0.5$.

(D) OPA1 $m R N A$ expression, and protein abundance (inset) over time-course experiment after synchronization in primary human skeletal muscle cells treated with siRNA targeting OPA1 $(\operatorname{siOPA1})(\mathrm{n}=6)$ compared to scramble $\operatorname{siRNA}(\mathrm{n}=6)$. 
(E-H) Primary human skeletal muscle cells treated with siRNA targeting OPA1 (n=6) (siOPA1). Molecular-clock genes BMAL1 (E), NPAS2 (F), PER2 (G), and PER3 (H). $\uparrow p<0.05$ overall difference siOPA1 vs. scramble. $* * * * p<0.0001, * p<0.05$ siOPA1 vs. Scramble at time point (2-way ANOVA). Genes are shown in synchronized cells and presented at each zeitgeber-time post serum shock.

(I) Protein abundance of electron transport complexes. Orange siRNA targeting OPA1. Oneway ANOVA, $\uparrow=$ Overall effect of siOPA1 $(p<0.05),{ }^{*} p<0.05$ effect of siOPA1.

Figure 7. siRNA depletion of OPA1 increases mitochondrial reactive oxygen species, and OPA1-mediated changes in clock-gene expression can be restored by resveratrol

(A) Schematic diagram of hypothesized mechanism.

(B) mRNA expression data from RNA sequencing of myotube cultures from donors with NGT or T2D ( $\mathrm{n}=7$, and 5, respectively) of HIF $1 \alpha$. Lines show the harmonic regression fits and solid line indicates circadian (adj.p<0.1) genes while dashed lines indicate non-circadian genes. Time points are hours post-synchronization.

(C) mRNA expression data from RNA sequencing of myotube cultures from donors with NGT or T2D ( $n=7$, and 5, respectively) of antioxidant enzymes, and those linked to NAD $/ \mathrm{NADH}^{+}$ metabolism that localize to mitochondria. Lines show the harmonic regression fits and solid line indicates circadian $(\operatorname{adj} . p<0.1)$ genes while dashed lines indicate non-circadian genes (Rain analysis). Time points are hours post-synchronization.

(D) $\mathrm{NAD}^{+}$concentration in synchronized primary human skeletal muscle cells $(n=6)$ treated with either scramble (blue) or siRNA targeting OPAl (orange), two-way ANOVA, foverall variance of time, $*<0.05$ difference between timepoints (at 12 vs 20 hours, and 28 vs 36 hours) only in scramble condition. 
(E) Primary human skeletal muscle cells treated either with scramble-scramble siRNA or siOPA1-siRNA targeting OPA1 (n=6), OPA1 gene expression. live-cell microscopy measuring MitoSOX fluorescence, indicative of mitochondrial ROS. OPA1 depleted myotubes had increased MitoSOX fluorescence perfused with Tyrode's solution, compared to scramble control. Resveratrol reduced ROS levels in cells with depleted OPA1 expression compared to siOPA1 and scramble control. Mixed effects-analysis, $\uparrow=p<0.05$ overall difference between conditions, $* * *=p<0.0001$ difference between siOPA1 vs siOPA1-resv, Scramble vs siOPA1, and Scramble vs siOPA1-resv.

(F and G) Gene expression of synchronized cells from men with NGT $(n=4)$ were treated with either scramble (blue) or siRNA targeting OPA1 (orange) at ZT28. Cells were either treated with $10 \mu \mathrm{M}$ Resveratrol or vehicle (control) for the duration of the experiment, subsequent to synchronization. Relative to scramble siRNA at ZT28. Control conditions are presented as a dotted line in the figures. Two-way ANOVA, $\uparrow=p<0.05$ overall difference, $* * p<0.01$ controlScramble vs. Control-treated siOPA1. ${ }^{* *} \mathrm{p}<0.001$ siOPA1 vs scramble. (F) OPA1 and (G) $N P A S 2$ gene expression. Results are mean \pm SEM.

\section{Supplementary Figure 1.}

Multi-dimensional scaling (MDS) plots showing dimensions 1-2 (left panels) and 2-3 (right panels) before and after patient batch effect removal. Each experimental condition is presented in A) Patient, B) Disease, C) Treatment and D) Time. Dimension 1 separates the disease group while dimension 3 separates time of sampling after removing the patient batch effect. Time is ZT hours.

\section{Supplemental Figure S2.}

Mitochondrial respiratory function (Mito Stress test, Seahorse XF analyzer) at ZT24 in synchronized myotubes from donors with normal glucose tolerance (NGT; $n=5)$ or type 2 
diabetes (T2D; $\mathrm{n}=5)$. 2-way repeated measures ANOVA of time-course. Solid lines are overall mean values, while dashed lines are means of individual donors.

\section{Supplementary Figure 3.}

mRNA expression data from RNA sequencing of myotube cultures from donors with NGT or T2D ( $\mathrm{n}=7$, and 5, respectively) of $H I G D 2 A, O M A 1$, and $O P A 1$. Lines show the harmonic regression fits and solid line indicates circadian $(\operatorname{adj} . p<0.1)$ genes while dashed lines indicate non-circadian genes (RAIN analysis). Time points are hours post-synchronization. Red=T2D, Black=NGT.

\section{Supplemental Table 1.}

Rhythmicity analysis results. Each group A) NGT (Control), B) T2D (Control), C) NGT (high glucose and insulin condition) and D) T2D (high glucose and insulin condition) was analyzed with RAIN using longitudinal method. Columns: Group, analysis group; ENSEMBL ID, gene ID from ENSEMBL database; Gene, gene name/symbol; phase and peaks.shape, phase \& peak shape estimates from RAIN; period, period of rhythmicity (24 hours); pVal, p-value; pVal.adj, p-values adjusted for multiple comparison by Benjamini-Hochberg method; peak, peak time (hours); trough, trough time (hours); amplitude and phase.estimate; amplitude and phase (peak time) calculated with mean centered data using harmonic regression; description, information about gene; chromosome-start-end-strand, genomic location of the gene.

\section{Supplemental Table 2.}

Differential rhythmicity analysis results. T2D was compared to NGT in A) Control and B) high concentration of glucose and insulin conditions. The analysis was performed using DODR algorithm. Columns: Contrast, comparisons made; ENSEMBL ID, gene ID from ENSEMBL database; Gene, gene name/symbol; robustDODR_pVal/pVal.adj, raw/adjusted p-values from robustDODR method; robustHarmScaleTest_pVal/ pVal.adj, raw/adjusted p-values from 
robustHarmScaleTest method; meta_pVal/pVal.adj, combined (meta) raw/adjusted p-values from robust (robustDODR and robustHarmScaleTest); log2FC(amplitude), log2-fold-change of the relative amplitude (T2D vs high concentration of glucose and insulin); description, information about gene; chromosome-start-end-strand, genomic location of the gene

\section{Supplemental Table 3.}

Amplitude and phase estimates were calculated using harmonic regression. Relative amplitudes and phases (peak times) of A) NGT (Control), B) T2D (Control), C) NGT (High glucose and insulin) and D) T2D (high glucose and insulin) groups. Log2-ratio (T2D vs. NGT) of relative amplitudes and phase differences are presented for E) Control and F) high glucose and insulin treatment group. The analysis was performed using HarmonicRegression $\mathrm{R}$ package. Columns: Group, disease-treatment group; ENSEMBL ID, gene ID from ENSEMBL database; Gene, gene name/symbol; hr.amplitude/hr.phase/hr.pVal/hr.qVal, relative amplitude/phase (peak time)/p-value/q-value calculated by harmonic regression; $\log 2$ (Amplitude), $\log 2$-fold change of the relative amplitude; delta(phase), phase difference between groups; description, information about gene; chromosome-start-end-strand, genomic location of the gene. 


\section{KEY RESOURCES TABLE}

\begin{tabular}{|c|c|c|}
\hline REAGENT or RESOURCE & SOURCE & IDENTIFIER \\
\hline \multicolumn{3}{|l|}{ Antibodies } \\
\hline Anti-OPA1 & $\begin{array}{l}\text { Cell Signaling } \\
\text { Technology (USA) }\end{array}$ & \#80471 \\
\hline Total OXPHOS & Abcam (UK) & \#110411 \\
\hline$\beta$-actin & Sigma-Aldrich (USA) & \#A5441 \\
\hline BMAL1 & Sigma-Aldrich (USA) & \#SAB4300614 \\
\hline CLOCK & Abcam (USA) & \#ab3517 \\
\hline \multicolumn{3}{|l|}{ Biological Samples } \\
\hline Human skeletal muscle biopsies & Karolinska Institutet & $\begin{array}{l}\text { Described in } \\
\text { methods }\end{array}$ \\
\hline Myo-Cre Opa1-/- mice & $\begin{array}{l}\text { University of } \\
\text { Barcelona }\end{array}$ & $\begin{array}{l}\text { Described in } \\
\text { methods }\end{array}$ \\
\hline C57BI/6j mice skeletal muscle & University of Florida & $\begin{array}{l}\text { Described in } \\
\text { methods }\end{array}$ \\
\hline \multicolumn{3}{|l|}{ Critical Commercial Assays } \\
\hline $\begin{array}{l}\text { Seahorse XF Cell } \\
\text { Mito Stress Test Kit }\end{array}$ & $\begin{array}{l}\text { Agilent Technologies, } \\
\text { USA }\end{array}$ & Kit 103015-100 \\
\hline \multicolumn{3}{|l|}{ Deposited Data } \\
\hline Raw and analyzed data & This paper & $\begin{array}{l}\text { GEO } \\
\text { https://www.ncbi.nlm } \\
\text {.nih.gov/geo/query/a } \\
\text { cc.cgi?\&acc=GSE14 } \\
\text { 3334, }\end{array}$ \\
\hline \multicolumn{3}{|l|}{ Experimental Models: Cell Lines } \\
\hline $\begin{array}{l}\text { Primary human skeletal muscle cells normal glucose } \\
\text { tolerant }\end{array}$ & Karolinska Institutet & $\begin{array}{l}\text { Described in } \\
\text { methods }\end{array}$ \\
\hline Primary human skeletal muscle cells Type 2 diabetes & Karolinska Institutet & $\begin{array}{l}\text { Described in } \\
\text { methods }\end{array}$ \\
\hline \multicolumn{3}{|l|}{ Oligonucleotides } \\
\hline Primers for RT-qPCR: see table 3 of methods & This paper & $\begin{array}{l}\text { Described in Table } 3 \\
\text { of methods }\end{array}$ \\
\hline \multicolumn{3}{|l|}{ Software and Algorithms } \\
\hline Prism: version 8 & Graphpad software & N.A. \\
\hline R: version 3.5 & N.A. & N.A. \\
\hline Python version 3.7 & N.A. & N.A. \\
\hline Microsoft Excel: version 2016 & Microsoft & N.A. \\
\hline FastQC (version 0.11.5) & $\begin{array}{l}\text { Babraham Institute, } \\
\text { UK }\end{array}$ & link \\
\hline Trim_Galore! (version 0.4.3) & $\begin{array}{l}\text { Babraham Institute, } \\
\text { UK }\end{array}$ & $\underline{\text { link }}$ \\
\hline
\end{tabular}




\begin{tabular}{|c|c|c|}
\hline STAR (version 2.5.3a) & Dobin et al., 2013 & $\begin{array}{l}\text { PMID: } 23104886 \\
\text { DOI: } \\
\text { 10.1093/bioinformati } \\
\text { cs/bts635 }\end{array}$ \\
\hline featureCounts (version 1.5.2) & Liao et al., 2014 & $\begin{array}{l}\text { PMID: } 24227677 \\
\text { DOI: } \\
\text { 10.1093/bioinformati } \\
\text { cs/btt656 }\end{array}$ \\
\hline multiQC (version 1.5) & Eewls et al., 2016 & $\begin{array}{l}\text { PMID: } 27312411 \\
\text { DOI: } \\
\text { 10.1093/bioinformati } \\
\text { cs/btw354 }\end{array}$ \\
\hline limma (version 3.42.2) & Ritchie et al., 2015 & $\begin{array}{l}\text { PMID: } 25605792 \\
\text { DOI: } \\
\text { 10.1093/nar/gkv007 }\end{array}$ \\
\hline DODR (version 0.99.3) & $\begin{array}{l}\text { Thaben \& } \\
\text { Westermark, } 2016\end{array}$ & $\begin{array}{l}\text { PMID: } 27207944, \\
\text { DOI: } 10.1093 / \text { bioinfo } \\
\text { rmatics/btw309 }\end{array}$ \\
\hline RAIN (version 1.20.0) & $\begin{array}{l}\text { Thaben \& } \\
\text { Westermark, } 2014\end{array}$ & $\begin{array}{l}\text { PMID: } 25326247, \\
\text { DOI: } \\
10.1177 / 074873041 \\
4553029\end{array}$ \\
\hline HarmonicRegression (version 1.0) & Lück et al., 2014 & $\begin{array}{l}\text { PMID: } 25373909 \\
\text { DOI: } \\
\text { 10.1016/j.celrep.201 } \\
4.09 .021\end{array}$ \\
\hline clusterProfiler (version 3.14.3) & Yu et al. 2012 & $\begin{array}{l}\text { PMID: } 22455463 \\
\text { DOI: } \\
10.1089 / o m i .2011 .0 \\
118 \\
\end{array}$ \\
\hline Other & & \\
\hline
\end{tabular}




\section{Disrupted circadian core-clock oscillations in Type 2 Diabetes are linked to altered rhythmic mitochondrial metabolism}

\section{Methods}

\section{Resource Availability}

\section{Lead Contact}

Further information and requests for resources and reagents should be directed to and will be facilitated by the Lead Contact, Juleen R. Zierath (Juleen.Zierath@ki.se).

\section{Materials Availability}

This study did not generate new unique reagents.

\section{Data and Code Availability}

The data and codes related to the findings of this study will be available from the corresponding author after publication upon reasonable request. The research team will provide an email address for communication once the data are approved to be shared with others. The proposal with detailed aims, statistical plan, and other information/materials may be required to guarantee the rationality of requirement and the security of the data. The patient-level data, but without names and other identifiers, will be shared after review and approval of the submitted proposal and any related requested materials.

\section{Human study methods}

\section{Study group and skeletal muscle biopsy procedure}

Study groups included individuals with normal glucose tolerance (NGT), as determined by oral glucose tolerance test (OGTT), or Type 2 diabetes (T2D). Written informed consent was obtained from all participants. The study protocol was approved by the regional ethics board in Stockholm, Sweden. The participants were instructed to refrain from physical exercise $48 \mathrm{hrs}$ before the visit. All investigations were performed in the morning after an overnight fast. Anthropometric measurements were taken, and blood samples were obtained for clinical 
chemistry analysis. After application of local anesthesia $(10 \mathrm{mg} / \mathrm{ml}$ mepivacaine hydrochloride, AstraZeneca, Cambridge, UK), a biopsy was obtained from m. vastus lateralis using a WeilBlakesley conchotome instrument (Agnthos, Sweden). Biopsies were cleared from any visible fat, connective tissue, or blood vessels and either used directly to prepare primary muscle cell cultures or immediately frozen in liquid nitrogen and stored at $-80^{\circ} \mathrm{C}$ until microarray analysis. The clinical characteristics of the NGT $(n=7)$ or T2D $(n=5)$ donors for the muscle cell culture studies are presented in Table 1.

\section{Cell culture}

Primary myoblasts from muscle biopsies from men with NGT and T2D were grown in DMEM/F12+Glutamax with 16\% FBS and 1\% Antibiotic-Antimycotic (100X). Differentiation was induced at $80 \%$ confluence, by incubating the cells for $4-5$ days in fusion medium consisting of 76\% DMEM Glutamax with $25 \mathrm{mmol} / \mathrm{l}$ glucose, 20\% M199 (5.5 mmol/l), 2\% HEPES and $1 \%$ Antibiotic-Antimycotic (100X), with $0.03 \mu \mathrm{g} / \mathrm{ml}$ zinc sulfate and $1.4 \mathrm{mg} / \mathrm{ml}$ Vitamin B12, with a further addition of $100 \mu \mathrm{g} / \mathrm{ml}$ apo-transferrin and $0.286 \mathrm{IU} / \mathrm{ml}$ insulin prior to use. Experiments were performed on differentiated myotubes after an additional 3-5 days incubation in the same media containing $2 \%$ FBS (post fusion media).

\section{Circadian experiments}

After post fusion, DMEM with $5.5 \mathrm{mmol} / 1$ glucose was used in the post fusion medium to create a low glucose condition. After 22 hrs, cells were synchronized by serum shock (media containing 50\% FBS, 2 hrs) (Balsalobre et al., 1998) and then switched back to low glucose media. Skeletal muscle myotubes from men with either T2D or NGT were lysed at 6-hr intervals from 12 hrs to $54 \mathrm{hrs}$. Zeit-time (ZT) was determined as time after synchronization.

\section{RNA-sequencing}

RNA was checked for quality using the Agilent RNA 600 nano kit and Bioanalyser instrument (Agilent Technologies, Santa Clara, CA). Aliquots of RNA (1 $\mu \mathrm{g})$ were analyzed using the 
Illumina TruSeq Stranded Total RNA with Ribo-Zero Gold protocol (Illumina) as described (Laker et al., 2017; Nylander et al., 2016). Ribosomal RNA was removed from the sample using $35 \mu 1$ rRNA removal beads (Illumina) on a magnetic plate followed by clean-up of the ribosomal-depleted RNA with $193 \mu$ l Agencourt RNAClean XP beads (Beckman Coulter), 70\% ethanol wash and elution into $10 \mu$ l Elution buffer (Illumina). The RNA sample was fragmented for $4 \mathrm{~min}$ at $94^{\circ} \mathrm{C}$ in Elute, Prime, Fragment High Mix (Illumina) and then subjected to first strand cDNA synthesis with $1 \mu 1$ Superscript III reverse transcriptase (Life Technologies) per sample using a thermocycler programmed to $25^{\circ} \mathrm{C}$ for $10 \mathrm{~min}, 50^{\circ} \mathrm{C}$ for $15 \mathrm{~min}$ and $70^{\circ} \mathrm{C}$ for 15 min. Second strand cDNA was synthesized by addition of Second Strand Marking Master Mix and samples were incubated at $16^{\circ} \mathrm{C}$ for $60 \mathrm{~min}$. Samples were subject to another bead clean up prior to A-tailing and ligation of adapters as per kit instructions (Illumina). Following a third bead clean-up, samples were enriched for DNA fragments by amplification using the Illumina PCR Primer Cocktail and PCR Master Mix, subjected to $98^{\circ} \mathrm{C}$ for $30 \mathrm{~min}$, followed by a pre-defined cycle $\left(98^{\circ} \mathrm{C}\right.$ for $10 \mathrm{~s}, 60^{\circ} \mathrm{C}$ for $30 \mathrm{~s}$ and $72^{\circ} \mathrm{C}$ for $\left.30 \mathrm{~s}\right)$ that was repeated $3-15$ times, based on each individual sample, and finally incubated for 5 min at $72^{\circ} \mathrm{C}$. Samples were cleaned, and validated for DNA concentration using the Qubit dsDNA HS assay kit (Invitrogen) and for base pair size and purity using the Agilent High Sensitivity DNA chip and Bioanalyser instrument. Libraries were subjected to 100-bp single-end sequencing on the X Ten platform (Illumina) at the Beijing Genomics Institute (BGI), Hong Kong, China. Approximately 20 million reads/sample were assigned to genes with 14,051 genes surviving the expression threshold.

\section{RNA-seq data analysis}

RNA-seq reads $(\bar{n} \approx 40 \mathrm{M})$ from FASTQ files were quality-trimmed using Trim_Galore (v0.4.3). Trimmed reads were aligned using STAR (v2.5.3a) (Dobin et al., 2013)aligner with Ensembl human annotation (GRCh38, release 92) (Zerbino et al., 2018) and gene features were 
counted using featureCounts from subread (v1.5.2) package (Liao et al., 2014) resulting in 27 M uniquely mapped and $20 \mathrm{M}$ assigned reads to genomic features (genes) on average, respectively. The lowly expressed genes were discarded from downstream analysis using filterByExpr function from edgeR package (Robinson et al., 2010) resulting 18,482 genes. As an apparent batch effect was introduced by participants (Figure S1), this batch effect was removed by using limma's removeBatchEffect function (Ritchie et al., 2015) for further downstream rhythmicity (RAIN) and differential rhythmicity (DODR) analysis as these tools do not consider any batch effect by default. The batch corrected CPM values were in $\log 2$ scale.

\section{Rhythmicity and differential rhythmicity analysis}

$p$ values for rhythmicity were assessed using RAIN (Thaben and Westermark, 2014) with longitudinal method and adjusted for multiple testing using Benjamini-Hochberg method. Genes with adjusted p-value below $0.10\left(\right.$ FDR RAIN $\left._{2} 0.10\right)$ were considered as rhythmic. We chose RAIN because this algorithm detects both symmetric and non-symmetric wave forms (Thaben and Westermark, 2014). Differential rhythmicity analysis was performed on mean centered $\log \mathrm{CPM}$ values (batch corrected) between myotubes from NGT and T2D donors (control and HGI group, separately) by using DODR (Thaben and Westermark, 2016) with "robust" method and resulting meta p-values were adjusted for multiple testing using Benjamini-Hochberg. It is essential to mean-center the $\log \mathrm{CPM}$ values as since DODR internally assumes identical means in order to test for differential rhythmicity. Genes with adjusted meta p-value below $0.10($ FDRDODR $<0.10)$ were considered differentially rhythmic.

\section{Circadian amplitude estimations}

A harmonic regression model using HarmonicRegression R package (Luck et al., 2014) with first degree polynomial component was fit to (participant) batch corrected logCPM values. As the calculated absolute amplitudes were in $\log 2$ scale $\left(A_{\log }\right)$, these amplitudes were transformed to relative amplitudes using the following equations. As fold-change amplitude $\left(A_{f c}\right)$ is 


$$
A_{f c}=\frac{1+A_{r e l}}{1-A_{r e l}}
$$

and twice the absolute amplitude calculated from $\log 2$ transformed data (batch corrected $\log \mathrm{CPM})$ equals to $\log 2$ of fold-change amplitude $\left(A_{f c}\right)$ :

$$
\text { 2. } A_{\log }=\log _{2}\left(A_{f c}\right)
$$

the relative amplitude (Arel) can be derived by solving these two equations to explain the relationship between $A_{\log }$ and $A_{\text {rel }}$ as

$$
A_{\text {rel }}=\frac{2^{2 \cdot A_{\log }-1}}{2^{2 \cdot A_{\log }+1}}
$$

To compare the relative amplitudes between T2Ds and NGTs, $\log 2$ ratios were calculated for each treatment group (control and HGI):

$$
\log _{2}\left(A_{r e l}\right)=\log _{2}\left(\frac{A_{r e l_{T 2 D}}}{A_{r e l_{N G T}}}\right)
$$

\section{Gene enrichment analysis}

Gene enrichment analysis was performed by using both over-representation analysis (ORA) and gene-set enrichment analysis (GSEA) throughout this study. For biological interpretation of the biological processes, we used Reactome pathways (Jassal et al., 2020) while gene ontology (GO) (Ashburner et al., 2000) cellular components (CC) was used in order to identify the enrichment of subcellular compartments. clusterProfiler (Yu et al., 2012) R package was used to analyze all gene enrichment results. Ontology terms (Reactome, GO:CC) with an adjusted p-value, using Benjamini-Hochberg method, below 0.10 were considered to be significantly enriched.

As we aimed to investigate the important biological pathways associated with the circadian genes, we subsetted circadian genes by disease-treatment groups (Figure 1D) and peak times (Figure 2F) and analyzed enrichment from Reactome pathways using ORA. Similarly, genes with significant correlations between insulin resistance (M-value) and basal 
gene expression (Figure 4C) were tested for GO:CC enrichment using ORA method. When a ranking metric, such as relative amplitude log2-fold-changes (Figure 4C), was preferred to analyze enrichment for cellular components, we used GSEA method with GO:CC database. An ontology-gene interaction network with top enriched (GSEA) GO:CC terms, so called CNET plot, was generated to show the involvement of an individual gene, together with the Spearman correlation coefficient (Figure 4D) and relative amplitude log2-fold-change (Figure 3B).

Lastly, clustering analysis revealed 10 distinct clusters from integrative analysis using binary input for presence/absence of rhythmicity in disease-treatment groups, insulin sensitivity correlation with basal gene expression and BMAL/CLOCK binding. The genes in each cluster were analyzed using GO:CC database with ORA method (Figure 5C), similar to the analysis performed in Figure 1D and 2F.

\section{Hyperinsulinemic euglycemic clamp procedure}

This study group was handled as described in the section "Human study methods". The clinical characteristics of the men with NGT $(n=22)$ or T2D $(n=22)$ enrolled in the gene array and hyperinsulinemic euglycemic clamp study are reported in Table 2 . After a 45 min rest, the study participants underwent a hyperinsulinemic euglycemic clamp as described (Andersson et al., 2014). Following an intravenous bolus dose of insulin $\left(1,600 \mathrm{mU} / \mathrm{m}^{2}\right.$ body surface area), insulin was infused intravenously at a rate of $40 \mathrm{mU} / \mathrm{m}^{2} / \mathrm{min}$ for $2 \mathrm{hrs}$ and a variable intravenous infusion of glucose $(200 \mathrm{mg} / \mathrm{ml})$ was used to maintain euglycemia between $81-99 \mathrm{mg} / \mathrm{dl}(4.5-$ $5.5 \mathrm{mmol} / \mathrm{l})$. The infusion rate of glucose during the last $60 \mathrm{~min}$ of the clamp, when insulin levels are in a steady state, was used to calculate whole-body glucose disposal rates (M-value).

\section{ChIP-sequencing}

\section{Tissue harvesting}

Animal procedures were conducted in accordance with institutional guidelines for the care and use of laboratory animals as approved by the University of Florida Institutional Animal Care 
and Use Committee. For the ChIPseq data, two replicate samples for BMAL1 and CLOCK were used. Each sample required pooling gastrocnemius muscle from 10 adult C57BL/6J male mice (Jackson Labs, Farmington, Connecticut, USA). The mice were entrained to $12 \mathrm{~L} / 12 \mathrm{D}$ schedule and all tissues were collected at ZT2 and frozen immediately.

\section{ChIP-Seq sample preparation}

For the CLOCK ChIPseq samples: Skeletal muscle was homogenized (muscle:buffer ratio 1:10) in lysis buffer (10 mM HEPES, pH7.5, $10 \mathrm{mM} \mathrm{MgCl}_{2}, 60 \mathrm{mM} \mathrm{KCl}, 300 \mathrm{mM}$ sucrose, $0.1 \mathrm{mM}$ EDTA, pH 8.0, 0.1\% Triton X-100, 1 mM DTT) with EGS (ethylene glycol bis (succininic acid) N-hydroxysuccinimide ester, Bio-world) (Koike et al., 2012). The reaction was stopped with 1 M Tris ( $\mathrm{pH}$ 7.5) buffer (final concentration of Tris was $20 \mathrm{mM}$ ) and formaldehyde added (final concentration of $1 \%$ ). Samples were incubated for $25 \mathrm{~min}$ at room temperature and crosslinking was stopped by adding glycine to $125 \mathrm{mM}$.

For the BMAL1 ChIPseq samples, we followed the protocol outlined in Hodge et al., 2019 (Hodge et al., 2019). Skeletal muscles were homogenized in lysis buffer containing 1\% of formaldehyde. Samples were incubated at room temperature for 25 min and crosslinking was stopped by adding glycine to $125 \mathrm{mM}$.

For all samples, myonuclei were isolated from skeletal muscle homogenates as described (Hodge et al., 2019) and samples were incubated with the appropriate antibody at $4{ }^{\circ} \mathrm{C}$ overnight. Samples were washed and then incubated at $65^{\circ} \mathrm{C}$ overnight to de-crosslink. Lastly, RNase, $5 \mathrm{M} \mathrm{NaCl}$ and Proteinase $\mathrm{K}$ were added, and samples were incubated at $55^{\circ} \mathrm{C}$ for $1 \mathrm{hr}$. DNA was recovered with a PCR purification kit (Qiagen) and the ChIPseq library was prepared by using NEBNext Ultra DNA library prep kit for Illumina. Library quality was determined by Tapestation and Quantitative PCR. Sequencing parameters were paired end $100 \mathrm{bp}$ run with approximately 40M reads/sample on the HiSeq 3000 Illumina sequencing system.

\section{ChIP-Seq data analysis}


The reads were aligned to Mus musculus genome assembly GRCm38 (mm 10) using Bowtie2 (Langmead and Salzberg, 2012) with the --sensitive-local option, which does not require that the entire read aligns from one end to the other. The biological replicates of the aligned reads were merged for CLOCK, BMAL1, and input, respectively. Homer software (Heinz et al., 2010) was deployed to perform peak calling for the CLOCK sample and the BMAL1 sample with the input sample as background. The default FDR rate threshold 0.001 was used to detect significant peaks.

Integrative analysis of circadian rhythmicity, insulin sensitivity and BMAL1/CLOCK chromatin binding

In order to identify the gene patterns across different experimental procedures, an integrative analysis approach was applied to circadian genes (RNAseq), insulin sensitivity correlations (Mvalue vs. basal gene expression from microarray study) and BMAL1/CLOCK chromatin binding (ChIP-seq). Both gene expression correlations with M-values and BMAL1/CLOCK ChIP-seq experiments were performed on mice, therefore we have identified the homologous genes between mouse and human and only included those genes for further downstream analysis. ChIP-seq peaks closest to a gene's transcription start site (TSS) are assigned to that gene. A binary matrix was created with the circadian rhythmicity (1: rhythmic, 0: nonrhythmic), Spearman correlation between insulin sensitivity (M-value) and basal gene expression (1: significant, 0: non-significant) and BMAL1/CLOCK binding (1: bound, 0: notbound). The resulting matrix was used for clustering analysis using "clust" algorithm (AbuJamous and Kelly, 2018) in order to identify groups of genes showing similar patterns. This resulted in 10 different clusters. Those 10 clusters were used for gene enrichment analysis using ORA method and GO:CC database.

The circadian genes associated with BMAL1/CLOCK binding were identified by overlapping each group, NGTs and T2Ds with both treatment conditions separately. The 
overlaps were tested by Fisher's exact test and resulting p-values were adjusted by BenjaminiHochberg method. Adjusted p-values below 0.05 were considered as statistically significant. Overlapping gene percentage, also known as Jaccard index, was used for showing the similarity between the overlaps (Figure 5A).

\section{Animal models}

\section{Myo-Cre Opa1 ${ }^{-/}$mice}

Animal work was performed in compliance with guidelines established by the University of Barcelona Committee on Animal Care. Opal ${ }^{\text {loxP/loxP }}$ mice were generated as reported (Ramirez et al., 2017; Rodriguez-Nuevo et al., 2018). The Myo-Cre OPAl mouse line was generated by crossing homozygous $O P A 1^{\text {loxP/loxP }}$ mice with a strain expressing Cre recombinase under the control of the myogenin promoter and the $1-\mathrm{kb}$ mouse $\mathrm{MEF} 2 \mathrm{C}$ enhancer, thus yielding a transgene called Myo-Cre (Li et al., 2005). Cre OPAl littermates were used as controls for knockout (KO) mice. Mice were kept under a 12-hr dark/light cycle and were provided standard chow diet and water ad libitum. Four-month-old male mice were used in all experiments. On the experimental day, mice were anesthetized with isoflurane, killed by cervical dislocation, and tibialis anterior muscles were harvested, and immediately frozen in liquid nitrogen for subsequent analysis.

\section{Mitochondrial disrupting compounds and circadian studies}

Primary human skeletal muscle cells from men with NGT were used for the study of the effect of compounds FCCP, Rotenone a, antimycin a, and oligomycin on molecular clock gene expression. Primary skeletal muscle cells were grown and differentiated as described above. A Lactate Dehydrogenase (LDH) assay was performed to determine cell viability after treatment with compounds, there were no differences in LDH concentration in any condition (data not shown). The cells were synchronized as described above and compounds or vehicle control were added at ZT14 after synchronization, and cells harvested after 4h exposure (ZT18). 
Compounds were Rotenone/Antimycin A $(0.38 \mu \mathrm{m})$, FCCP $(2 \mu \mathrm{m})$ and Oligomycin $(1 \mu \mathrm{m})$.

Cells were harvested to collect RNA and core-clock gene mRNA expression was measured by RT-qPCT.

\section{Gene silencing and metabolic studies}

\section{Opa1 silencing}

Primary human skeletal muscle cells from men with NGT were used for the study of OPAI silencing. Primary skeletal muscle cells were grown and differentiated as described above. After switching to post fusion media, cells were transfected as described (Lassiter et al., 2018), using $5 \mu \mathrm{M}$ of either Silencer ${ }^{\circledR}$ Select (Thermo Fisher Scientific, Waltham, MA) Negative Control No. 2 (\#4390847) or validated siRNAs against OPA1 (\#s9852), for two separate 5-hr transfection periods separated by $\sim 48 \mathrm{hrs}$.

\section{Metabolic phenotyping of cells}

To assess the mitochondrial function of primary human skeletal muscle cells, cells were subjected to a Seahorse XF Mito Stress Test using the manufacturer's instructions for timing (Agilent, Santa Clara, CA), as described (Lassiter et al., 2018). Oxygen consumption rates (OCR) and extracellular acidification rates (ECAR) were measured at three time points under unstimulated conditions, then after treatment with $1 \mu \mathrm{M}$ oligomycin, $2 \mu \mathrm{M} \mathrm{FCCP}$, and $0.75 \mu \mathrm{M}$ rotenone + antimycin A. OCR and ECAR was normalized to the protein concentration, as quantified by a Pierce BCA protein assay kit (Thermo Fisher Scientific). Myotube cultures either from men with NGT $(n=5)$ or T2D $(n=5)$ were subjected to a Mito Stress (Figure S1) test under the same conditions as previously described, except, these cells were measured at ZT24 after synchronization as described in 'Circadian experiments' section. For circadian Seahorse XF experiments (time-course of basal OCR, Figure 3D), myotube cultures either from men with NGT $(n=5)$ or T2D $(n=5)$, were synchronized as described in "Circadian experiments" section. Myotubes were measured sequentially, i.e. the same cells were measured at all time points in 
buffered media, and only basal oxygen consumption was measured. This allowed minimal disturbance to cells, and enabled measurements of diurnal oxygen consumption in a method derived from previous work (Peek et al., 2013).

\section{NAD measurements of cells after OPA1 silencing}

NAD levels were assessed in primary human skeletal muscle cells after OPA1 silencing using an enzymatic cycling assay (Dall et al., 2018). Cells were harvested with trypsin, and the cell pellet was dissolved in $200 \mu \mathrm{l}$ of $0.6 \mathrm{M}$ perchloric acid and subjected to centrifugation for 3 mins at $13,000 \mathrm{~g}$. The supernatants were transferred to new tubes and diluted 1:100 in $100 \mathrm{mM}$ $\mathrm{Na}_{2} \mathrm{HPO}_{4}(\mathrm{pH} 8)$. An aliquot $(100 \mu \mathrm{l})$ of the diluted extract was pipetted into a 96-well plate, and a reaction mix $(100 \mu \mathrm{l})$ containing $100 \mathrm{mM} \mathrm{Na}_{2} \mathrm{HPO}_{4}, 10 \mu \mathrm{M}$ flavinmononucleotide, $2 \%$ ethanol, $90 \mathrm{U} / \mathrm{ml}$ alcohol dehydrogenase, $130 \mathrm{mU} / \mathrm{ml}$ diaphorase, $2.5 \mu \mathrm{g} / \mathrm{ml}$ resazurin and 10 $\mathrm{mM}$ nicotinamide was added. Fluorescence (Ex $540 \mathrm{~nm} / \mathrm{Em}$ 580) was measured over $30 \mathrm{~min}$ and the NAD content was calculated from a standard curve and normalized to the protein concentration. Protein was extracted by dissolving the cell pellet (after perchloric acid precipitation) in $200 \mu 10.2 \mathrm{M} \mathrm{NaOH}$, incubating at $95^{\circ} \mathrm{C}$ for $5 \mathrm{~min}$, and centrifugation for 5 mins at $13,000 \mathrm{~g}$. Protein concentration was determined on the supernatant using the Bicinchoninic Acid Assay (23227, Thermo Fisher Scientific, MA).

\section{Resveratrol treatment}

Human primary skeletal muscle cells were grown, differentiated and synchronized as described above. Cells were either treated with $10 \mu \mathrm{M}$ resveratrol (\#R5010, Merck KGaA, Darmstadt, Germany) or vehicle control for the duration of the experiment, subsequent to synchronization.

\section{Gene expression analysis}

Microarray analysis

RNA quality was assessed and ensured using the Experion ${ }^{\mathrm{TM}}$ Automated Electrophoresis System (Bio-Rad Laboratories, CA). Affymetrix GeneChip ${ }^{\mathrm{TM}}$ Human Transcriptome Array 2.0 
(Thermo Fisher Scientific, MA) was used for the whole-transcriptome analysis performed at the Bioinformatics and Expression Core facility (BEA) at Karolinska Institutet, Huddinge, Sweden. Amplified and biotinylated sense-strand DNA targets were generated from total RNA using the WT Plus Kit (Thermo Fisher, MA). Fragmented and biotinylated sense-strand DNA target $(5.5 \mu \mathrm{g})$ were hybridized to the arrays in GeneChip Hybridization Oven 645 at $45^{\circ} \mathrm{C}$ for 16-18 hrs. Arrays were washed and stained in GeneChip Fluidics Station 450 prior to scanning in Affymetrix GeneChip Scanner.

Gene array data were analyzed with packages available from Bioconductor (http://www.bioconductor.org). Normalization and calculation of gene expression was performed with the Robust Multichip Average expression measure using oligo package (Carvalho and Irizarry, 2010). Prior to further analysis, a nonspecific filter was applied to include genes with expression signal $>30$ in at least $25 \%$ of all samples Differentially expressed genes between NGT and T2D were identified using limma (Ritchie et al., 2015). The correlation between the basal gene expression and insulin sensitivity (M-value) was calculated by Spearman's rank correlation and p-values are adjusted for multiple testing using BenjaminiHochberg method. Spearman correlation coefficients were used for gene enrichment analysis using GSEA method with GO:CC database (See section "Gene enrichment analysis" for details). Ontology-gene interaction network, so called CNET plot, from this analysis using the top enriched terms are shown in Figure 4D. The color code represents the log2-fold-change of gene expression between NGT compared to T2D.

\section{PCR-analysis}

RNA was prepared from human skeletal muscle biopsies and primary human skeletal muscle cells. Gene expression was determined by using Fast SYBR Green Master Mix (Thermo Fisher Scientific) and self-designed oligonucleotides (oligonucleotide sequences provided below) or Predesigned TaqMan Gene Expression Assays (Thermo Fisher Scientific). SYBR human 
housekeeping: $B 2 M, G U S B, T B P, R P L O$. TaqMan mouse housekeeping genes: $B 2 m$ and

Gapdh, human: TBP, B2M. Primer sequences and assay IDs presented in Table 3.

\section{Immunoblot analysis}

Immunoblot analysis was performed as described (Lassiter et al., 2018) with antibodies against OPA1 (\#80471) from Cell Signaling Technology, Total OXPHOS (\#110411) from Abcam (UK) and $\beta$-actin (\#A5441) from Sigma-Aldrich. Protein abundance was normalized to $\beta$-actin.

\section{Live-cell microscopy and determination of mitochondrial ROS}

Primary human skeletal muscle cells from men with NGT were used for the determination of mitochondrial ROS by live-cell microscopy. Primary skeletal muscle cells were grown and differentiated as described above. MitoSOX (50 $\mu \mathrm{g}$, Thermo Fisher: M36008) was dissolved in $50 \mu 1$ pluoronic F127 (Invitrogen: P6866) and $50 \mu \mathrm{l}$ DMSO to make the MitoSOX stock solution. MitoSOX stock solution $(5 \mu \mathrm{l})$ was added to the media and incubated for $15 \mathrm{~min}$ at $37^{\circ} \mathrm{C}$ and $5 \% \mathrm{CO}_{2}$. Thereafter, media from the cell dish were removed, the dish was mounted on the confocal microscope (Zeiss LSM710) and perfused with Tyrode solution (in mM): 121 $\mathrm{NaCl}, 5 \mathrm{KCl}, 1.8 \mathrm{CaCl}_{2}, 0.4 \mathrm{NaH}_{2} \mathrm{PO}_{4}, 0.5 \mathrm{MgCl} 2,24 \mathrm{NaHCO}_{3}, 0.1 \mathrm{EDTA}$, and 5.5 glucose. Cells were then imaged with 2 mins interval for $20 \mathrm{~min}$ and thereafter switched to Tyrode solution supplemented with FCCP $(2 \mu \mathrm{M})$ for $20 \mathrm{~min}$. All images were analyzed with Image J.

\section{References}

Abu-Jamous, B., and Kelly, S. (2018). Clust: automatic extraction of optimal co-expressed gene clusters from gene expression data. Genome Biology 19, 172-172.

Andersson, D.P., Thorell, A., Lofgren, P., Wiren, M., Toft, E., Qvisth, V., Riserus, U., Berglund, L., Naslund, E., Bringman, S., et al. (2014). Omentectomy in addition to gastric bypass surgery and influence on insulin sensitivity: a randomized double blind controlled trial. Clin Nutr 33, 991-996.

Ashburner, M., Ball, C.A., Blake, J.A., Botstein, D., Butler, H., Cherry, J.M., Davis, A.P., Dolinski, K., Dwight, S.S., Eppig, J.T., et al. (2000). Gene ontology: tool for the unification of biology. The Gene Ontology Consortium. Nature Genetics 25, 25-29.

Balsalobre, A., Damiola, F., and Schibler, U. (1998). A serum shock induces circadian gene expression in mammalian tissue culture cells. Cell 93, 929-937. 
Carvalho, B.S., and Irizarry, R.A. (2010). A framework for oligonucleotide microarray preprocessing. Bioinformatics 26, 2363-2367.

Dall, M., Penke, M., Sulek, K., Matz-Soja, M., Holst, B., Garten, A., Kiess, W., and Treebak, J.T. (2018). Hepatic NAD $(+)$ levels and NAMPT abundance are unaffected during prolonged high-fat diet consumption in C57BL/6JBomTac mice. Mol Cell Endocrinol 473, 245-256.

Dobin, A., Davis, C.A., Schlesinger, F., Drenkow, J., Zaleski, C., Jha, S., Batut, P., Chaisson, M., and Gingeras, T.R. (2013). STAR: ultrafast universal RNA-seq aligner. Bioinformatics (Oxford, England) 29, 15-21.

Heinz, S., Benner, C., Spann, N., Bertolino, E., Lin, Y.C., Laslo, P., Cheng, J.X., Murre, C., Singh, H., and Glass, C.K. (2010). Simple combinations of lineage-determining transcription factors prime cis-regulatory elements required for macrophage and B cell identities. Mol Cell 38, 576-589.

Hodge, B.A., Zhang, X., Gutierrez-Monreal, M.A., Cao, Y., Hammers, D.W., Yao, Z., Wolff, C.A., Du, P., Kemler, D., Judge, A.R., et al. (2019). MYOD1 functions as a clock amplifier as well as a critical co-factor for downstream circadian gene expression in muscle. Elife 8, e43017.

Jassal, B., Matthews, L., Viteri, G., Gong, C., Lorente, P., Fabregat, A., Sidiropoulos, K., Cook, J., Gillespie, M., Haw, R., et al. (2020). The reactome pathway knowledgebase. Nucleic Acids Res 48, D498-d503.

Koike, N., Yoo, S.H., Huang, H.C., Kumar, V., Lee, C., Kim, T.K., and Takahashi, J.S. (2012). Transcriptional architecture and chromatin landscape of the core circadian clock in mammals. Science 338, 349-354.

Laker, R.C., Garde, C., Camera, D.M., Smiles, W.J., Zierath, J.R., Hawley, J.A., and Barres, R. (2017). Transcriptomic and epigenetic responses to short-term nutrient-exercise stress in humans. Sci Rep 7, 15134.

Langmead, B., and Salzberg, S.L. (2012). Fast gapped-read alignment with Bowtie 2. Nat Methods 9, 357-359.

Lassiter, D.G., Sjogren, R.J.O., Gabriel, B.M., Krook, A., and Zierath, J.R. (2018). AMPK activation negatively regulates GDAP1, which influences metabolic processes and circadian gene expression in skeletal muscle. Mol Metab 16, 12-23.

Li, S., Czubryt, M.P., McAnally, J., Bassel-Duby, R., Richardson, J.A., Wiebel, F.F., Nordheim, A., and Olson, E.N. (2005). Requirement for serum response factor for skeletal muscle growth and maturation revealed by tissue-specific gene deletion in mice. Proc Natl Acad Sci U S A 102, 1082-1087.

Liao, Y., Smyth, G.K., and Shi, W. (2014). featureCounts: an efficient general purpose program for assigning sequence reads to genomic features. Bioinformatics 30, 923930.

Luck, S., Thurley, K., Thaben, P.F., and Westermark, P.O. (2014). Rhythmic degradation explains and unifies circadian transcriptome and proteome data. Cell Rep 9, 741-751.

Nylander, V., Ingerslev, L.R., Andersen, E., Fabre, O., Garde, C., Rasmussen, M., Citirikkaya, K., Baek, J., Christensen, G.L., Aznar, M., et al. (2016). Ionizing radiation potentiates high-fat diet-induced insulin resistance and reprograms skeletal muscle and adipose progenitor cells. Diabetes 65, 3573-3584.

Peek, C.B., Affinati, A.H., Ramsey, K.M., Kuo, H.Y., Yu, W., Sena, L.A., Ilkayeva, O., Marcheva, B., Kobayashi, Y., Omura, C., et al. (2013). Circadian clock NAD+ cycle drives mitochondrial oxidative metabolism in mice. Science 342, 1243417.

Ramirez, S., Gomez-Valades, A.G., Schneeberger, M., Varela, L., Haddad-Tovolli, R., Altirriba, J., Noguera, E., Drougard, A., Flores-Martinez, A., Imbernon, M., et al. 
(2017). Mitochondrial dynamics mediated by mitofusin 1 is required for POMC neuron glucose-sensing and insulin release control. Cell Metab 25, 1390-1399 e1396.

Ritchie, M.E., Phipson, B., Wu, D., Hu, Y., Law, C.W., Shi, W., and Smyth, G.K. (2015). limma powers differential expression analyses for RNA-sequencing and microarray studies. Nucleic Acids Research 43, e47-e47.

Robinson, M.D., McCarthy, D.J., and Smyth, G.K. (2010). edgeR: a Bioconductor package for differential expression analysis of digital gene expression data. Bioinformatics 26 , 139-140.

Rodriguez-Nuevo, A., Diaz-Ramos, A., Noguera, E., Diaz-Saez, F., Duran, X., Munoz, J.P., Romero, M., Plana, N., Sebastian, D., Tezze, C., et al. (2018). Mitochondrial DNA and TLR9 drive muscle inflammation upon Opal deficiency. EMBO J 37.

Thaben, P.F., and Westermark, P.O. (2014). Detecting rhythms in time series with RAIN. J Biol Rhythms 29, 391-400.

Thaben, P.F., and Westermark, P.O. (2016). Differential rhythmicity: detecting altered rhythmicity in biological data. Bioinformatics 32, 2800-2808.

Yu, G., Wang, L.G., Han, Y., and He, Q.Y. (2012). clusterProfiler: an R Package for Comparing Biological Themes Among Gene Clusters. OMICS 16, 284-287.

Zerbino, D.R., Achuthan, P., Akanni, W., Amode, M.R., Barrell, D., Bhai, J., Billis, K., Cummins, C., Gall, A., Girón, C.G., et al. (2018). Ensembl 2018. Nucleic Acids Research 46, D754-D761. 
Table 1. Clinical Characteristics of the Donors for Muscle Cell Culture Studies.

\begin{tabular}{|c|c|c|c|c|c|}
\hline & \multicolumn{2}{|c|}{ NGT } & \multicolumn{2}{|c|}{ T2D } & \multirow[b]{2}{*}{ p-value } \\
\hline & Mean & SEM & Mean & SEM & \\
\hline Age & 62.6 & 1.1 & 60 & 1.4 & 0.005 \\
\hline Weight [kg] & 93.7 & 3.6 & 89.7 & 6.3 & 0.174 \\
\hline Height [cm] & 180.3 & 1.9 & 180.0 & 3.6 & 0.875 \\
\hline BMI $\left[\mathrm{kg} / \mathrm{m}^{2}\right]$ & 28.8 & 0.8 & 27.6 & 1.2 & 0.012 \\
\hline BP Systolic [mm Hg] & 139.6 & 3.3 & 138.2 & 8.9 & 0.716 \\
\hline BP Diastolic [mm Hg] & 87.9 & 3.0 & 81.5 & 5.4 & 0.022 \\
\hline Pulse [BPM] & 61.5 & 4.5 & 65.3 & 4.1 & 0.099 \\
\hline HbA1c $[\mathrm{mmol} / \mathrm{mol}]$ & 30.3 & 1.5 & 59.6 & 5.6 & $<0.0001$ \\
\hline Plasma-glucose 0 OGTT [mmol/l] & 5.14 & 0.20 & 9.06 & 0.85 & $<0.0001$ \\
\hline Plasma-glucose 120 OGTT [mmol/l] & 5.96 & 0.67 & 16.16 & 1.39 & $<0.0001$ \\
\hline Plasma-TG [mmol/l] & 1.41 & 0.38 & 1.25 & 0.26 & 0.084 \\
\hline Plasma-Chol [mmol/l] & 5.83 & 0.37 & 3.84 & 0.36 & $<0.0001$ \\
\hline Plasma-HDL [mmol/l] & 1.37 & 0.16 & 1.22 & 0.08 & $<0.0001$ \\
\hline Plasma-LDL [mmol/l] & 3.83 & 0.35 & 2.00 & 0.25 & $<0.0001$ \\
\hline $\mathrm{VO}_{2} \mathbf{m a x}[\mathrm{ml} / \mathrm{min}]$ & 2478 & 207 & 2735 & 361 & 0.194 \\
\hline $\mathrm{VO}_{2} \max [\mathrm{ml} / \mathrm{kg} / \mathrm{min}]$ & 28.7 & 2.0 & 31.6 & 2.6 & 0.153 \\
\hline Max workload [Watt] & 181.9 & 16.8 & 193.8 & 26.3 & 0.454 \\
\hline
\end{tabular}

Skeletal muscle biopsies were obtained from Normal Glucose Tolerant (NGT; $n=7)$ and Type 2 Diabetic (T2D; $\mathrm{n}=5$ ) men and myotube cultures were derived for in vitro studies. Results are mean \pm SEM. P-values were determined by Student's $t$-test. 
Table 2. Clinical Characteristics of the Participants in the Hyperinsulinemic Euglycemic Clamp Study.

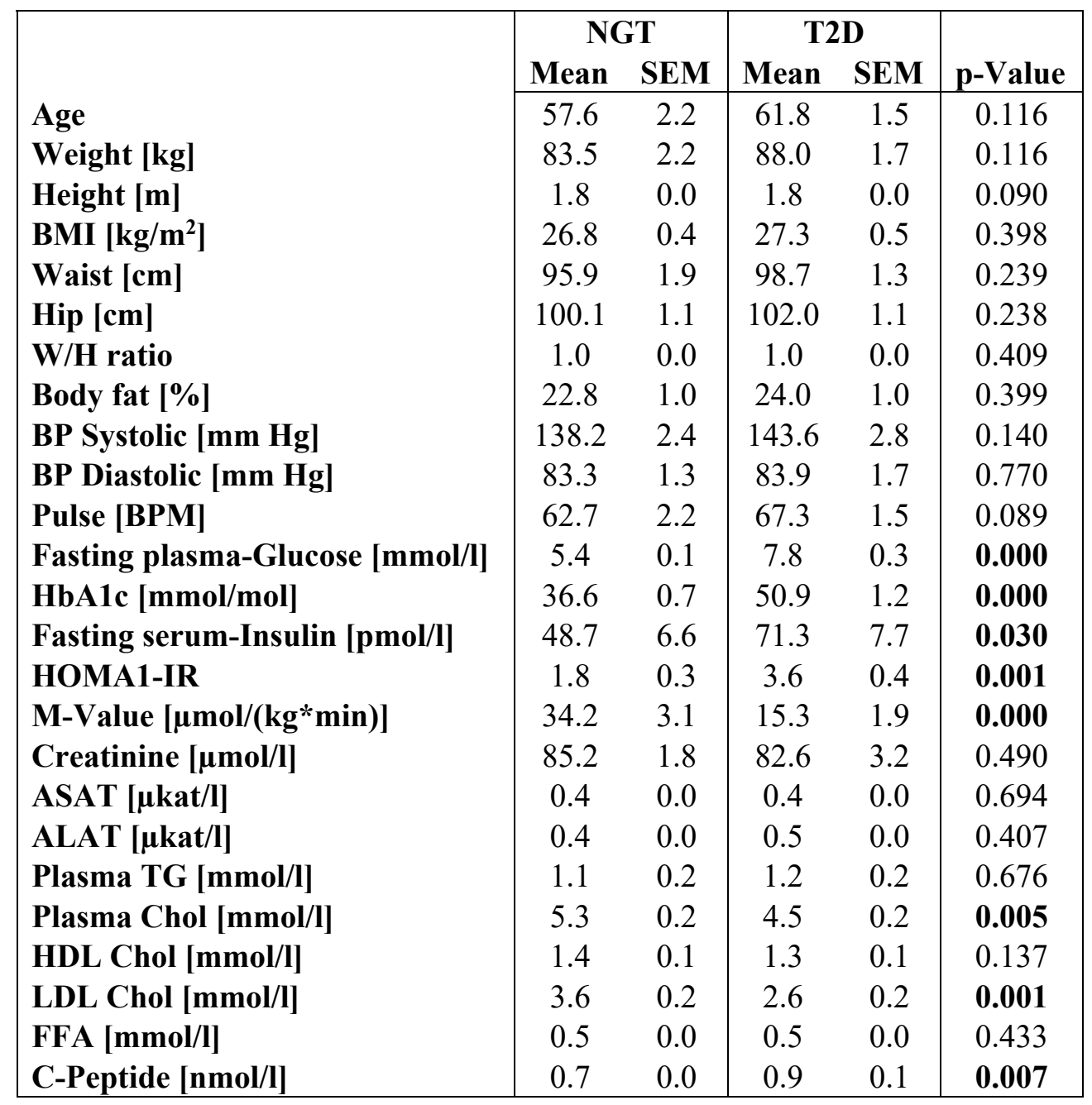

The study groups include men with normal glucose tolerance (NGT; $n=25)$, as determined by oral glucose tolerance test, or T2D $(n=25)$. Skeletal muscle biopsies were obtained for gene expression analysis. Insulin sensitivity (M-value) was determined by the hyperinsulinemic euglycemic clamp. Results are mean \pm SEM. P-values were determined by Student's $t$-test. 
Table 3. Primer Sequences and Assay IDs used for the Gene Expression Analysis.

\begin{tabular}{|c|c|c|}
\hline \multicolumn{3}{|c|}{ SYBR $^{\mathrm{TM}}$ Human } \\
\hline Gene & Sequ & ence \\
\hline \multirow{2}{*}{$A R N T L$} & Fwd & ACCACAAGAACTTCTAGGCAC \\
\hline & Rev & CCAGGACGTTGGCTAAAACA \\
\hline \multirow{2}{*}{ NPAS2 } & Fwd & TGAATCTGACCACACCTGCT \\
\hline & Rev & CTCTGGGCGTACTTGACTTG \\
\hline \multirow{2}{*}{ NR1D1 } & Fwd & CTGGGAGGATTTCTCCATGA \\
\hline & Rev & TCACTGTCTGGTCCTTCACG \\
\hline \multirow{2}{*}{ PER2 } & Fwd & CGTGTCAGTGTCCGGAAAAG \\
\hline & Rev & GGAGGAATTCTAGGGGCTTCA \\
\hline \multirow{2}{*}{$B 2 M$} & Fwd & ATATAAGTGGAGGCGTCGCG \\
\hline & Rev & TGAATCTTTGGAGTACGCTGG \\
\hline \multirow{2}{*}{ GUSB } & Fwd & GCAGATGTGTGACCGCTATG \\
\hline & Rev & TGAGCGATCACCATCTTCAAG \\
\hline \multirow{2}{*}{ TBP } & Fwd & AGTTCTGGGATTGTACCGCA \\
\hline & Rev & TATATTCGGCGTTTCGGGCA \\
\hline \multirow{2}{*}{ RPLO } & Fwd & TGGAGAAACTGCTGCCTCAT \\
\hline & Rev & GATTTCAATGGTGCCCCTGG \\
\hline
\end{tabular}

\begin{tabular}{|ll|}
\hline \multicolumn{2}{|l|}{ TaqMan } \\
\hline Gene & Assay ID \\
\hline \multicolumn{2}{|c|}{ Mouse } \\
\hline B2m & Mm00437762_m1 \\
Gapdh & Mm99999915_g1 \\
Opa1 & Mm01349707_g1 \\
Arntl & Mm00500226_m1 \\
Clock & Mm00455950_m1 \\
Nr1d1 & Mm00520708_m1 \\
Per2 & Mm00478099_m1 \\
Per3 & Mm00478120_m1 \\
\hline & Human \\
\hline OPA1 & Hs01047018_m1 \\
TBP & Hs00427620_m1 \\
B2M & Hs00187842_m1 \\
PER3 & Hs00213466_m1 \\
\hline
\end{tabular}


A

Primary cell culture Circadian experiment RNA-Sequencing

bioRxiv preprint doi: https://ld ord/10 $1101 / 2021,02.24$ (which was not certified by peer review) is the aut

available under aCC-BY-NC-ND 4.0 International license.

\section{Skeletal muscle biopsies}

Type 2 Diabetic \& Normal

glucose tolerant subjects

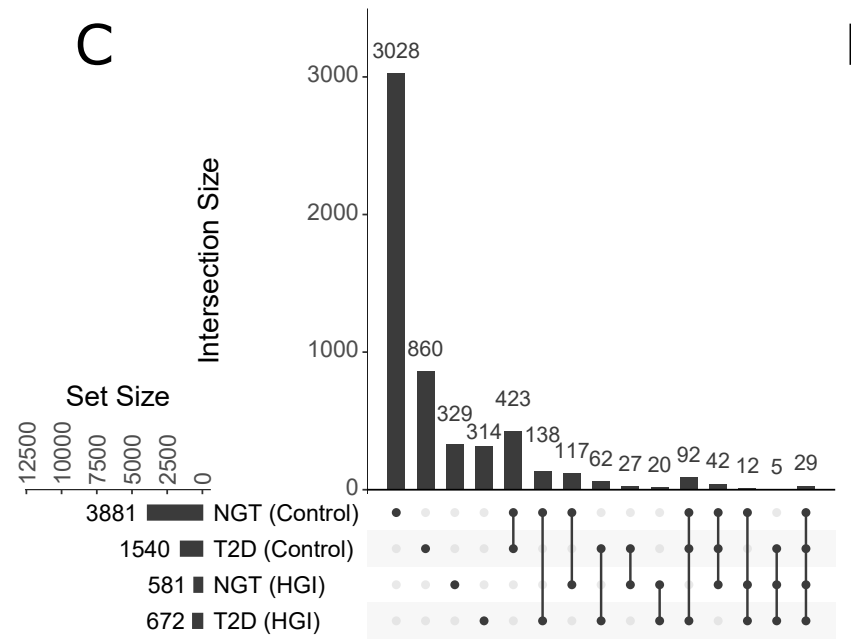

$\mathrm{D}$

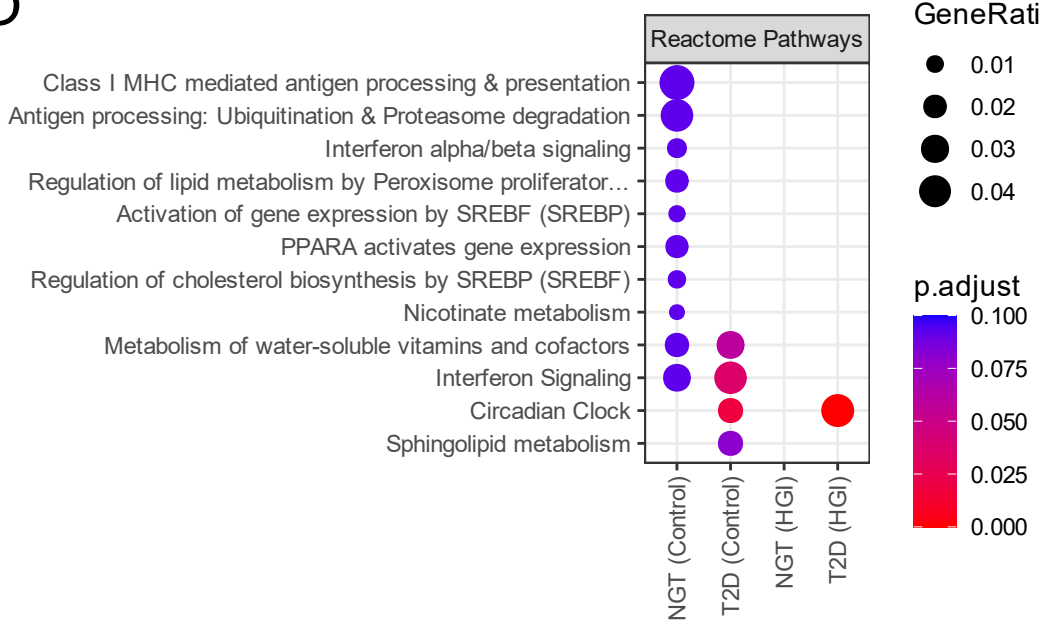

$E$

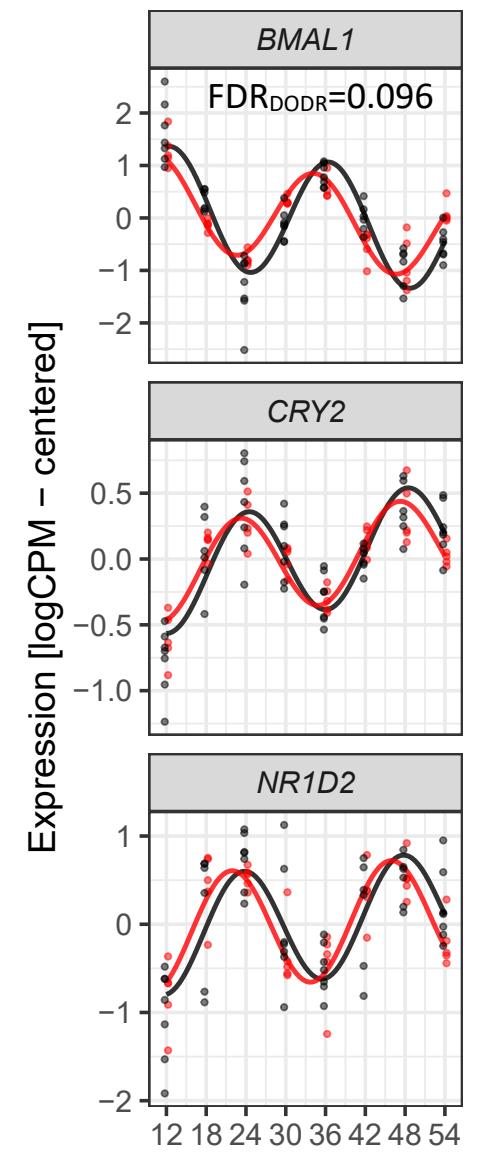

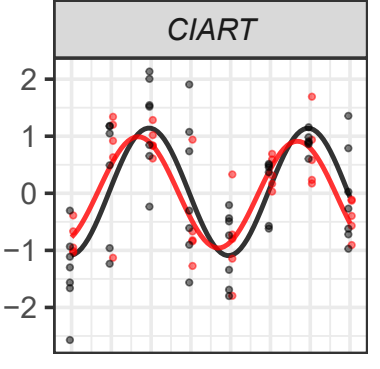
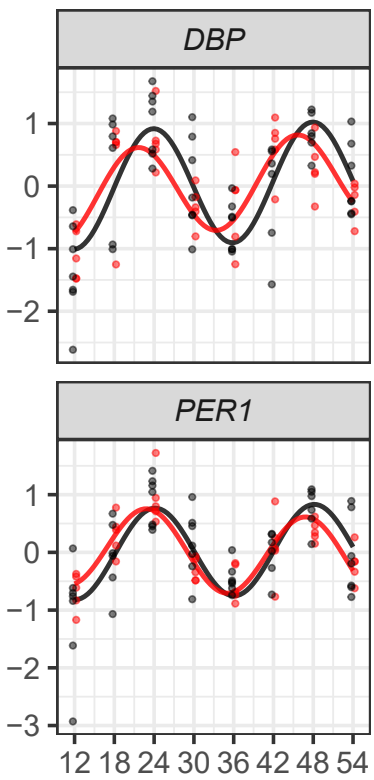
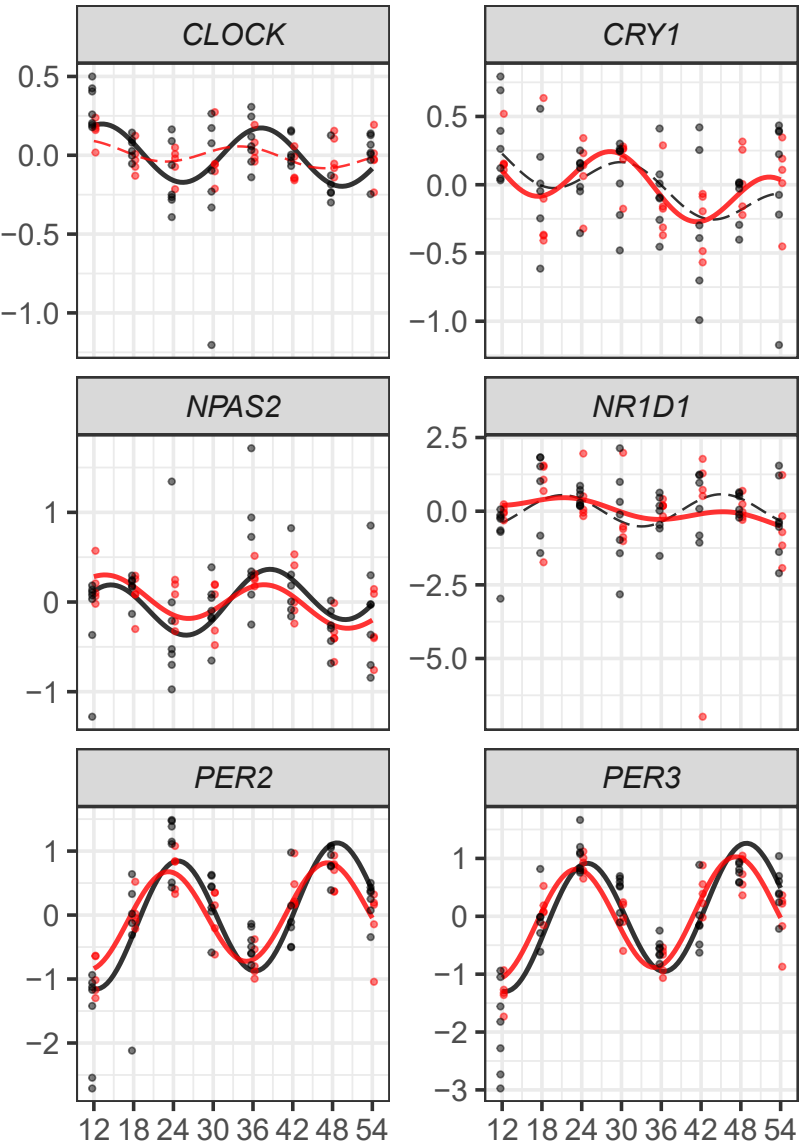

group: $\rightarrow$ NGT $\rightarrow$ T2D

rhythmic: -- YES - NO

Figure 1 

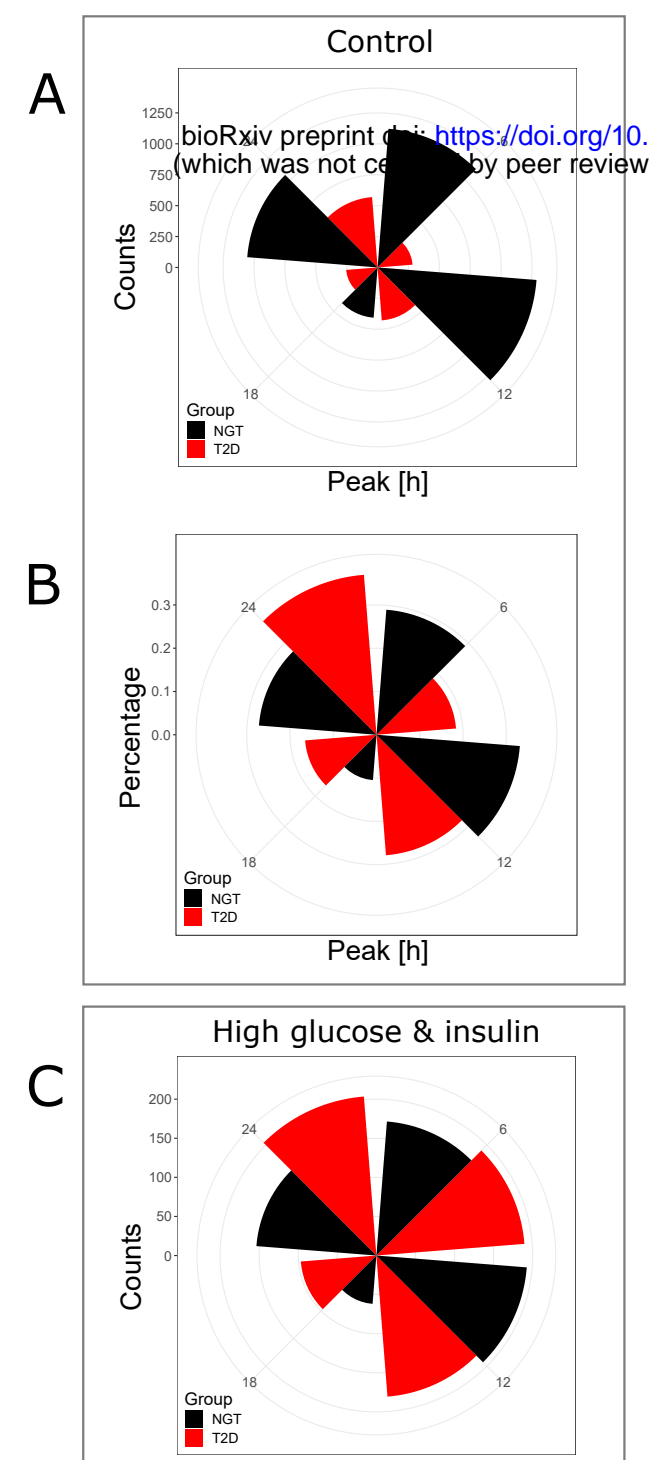

Peak [h]

D

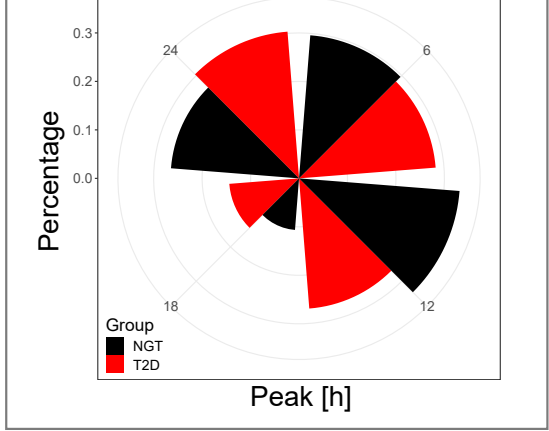

$\mathrm{E}$ the author/fund available under aCC-
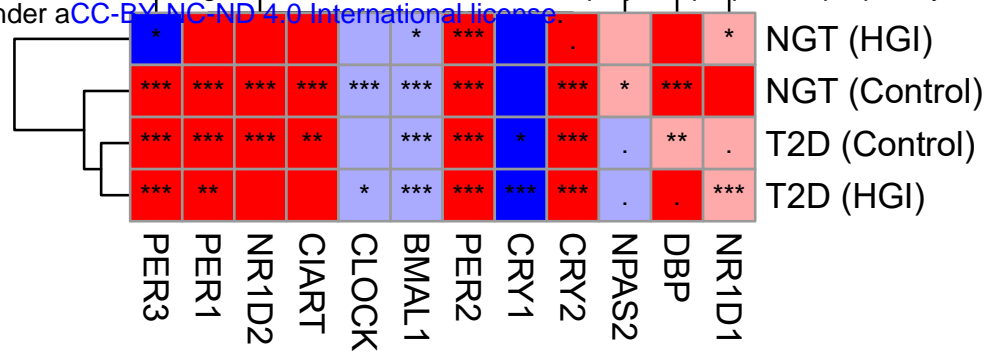

$\mathrm{F}$

Sphingolipid metabolism Mr transcription pathway
Mitotic Prometaphase Cilium Assembly -

Cyclin A:Cdk2-associated events at $S$ phase entry Cyclin $\mathrm{E}$ associated events dung $\mathrm{G} / \mathrm{S}$ transition Phase 0 - rapid depolarisation
Interaction between $\mathrm{L} 1$ and Ankyrins Cardiac conduction Interferon Signaling nterferon alpha/beta signaling Metabolism of carbohydrates Hyaluronan uptake and degradation Antiviral mechanism by IFN-stimulated genes BMAL1:CLOCK,NPAS2 activates circadian gene expression PPARA activates gene expression
Regulation of cholesterol biosynthesis by SREBP (SREBF) Activation of gene expression by SREBF (SREBP) Regulation of lipid metabolism by Peroxisome proliferator... ransport of Mature mRNA derived from an Intronar receptors Cleavage of Growing Transcript in the Termination Region mRNA Splicing - Major Pathway mRNA Splicing -

Processing of Capped Intron-Containing Pre-mRNA Polymerase II Transcription Termination Caspase activation via extrinsic apoptotic signalling pat... Diseases associated wiseases of Immune System with the TLR signaling cascade Eukaryotic Translation Initiation -
13a-mediated translational silencing of Ceruloplasmin ex... Cap-dependention ap-dependent Translation Initiation Peptide chain elongation Eukaryotic Translation Elongation Selenoamino acid metabolion Selenocysteine synthesis

Formation of a pool of free 40 S subunits NMD) indotic Translation Termination Nonsense Mediated Decay (NMD) independent of the Exon Jun...
PRC2 methylates histones and DNA
Packaging Of Telomere Ends
Condensation of Prophase Chromosomes Nonsense Mediated Decay (NMD) independent of the Exon Jun...
PRC2 methylates histones and DNA
Packaging Of Telomere Ends
Condensation of Prophase Chromosomes Nonsense Mediated Decay (NMD) independent of the Exon Jun...
PRC2 methylates histones and DNA
Packaging Of Telomere Ends
Condensation of Prophase Chromosomes DNA methylation

Activated PKN1 stimulates transcription of AR (androgen $r$... Nonhomologous End-Joining (NHEJ) RNA Polymerase I Promoter Opening Nucleosome assembly
GeneRatio

- 0.025

0.050

0.075

0.100

p.adjust

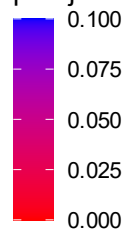

Figure 2 


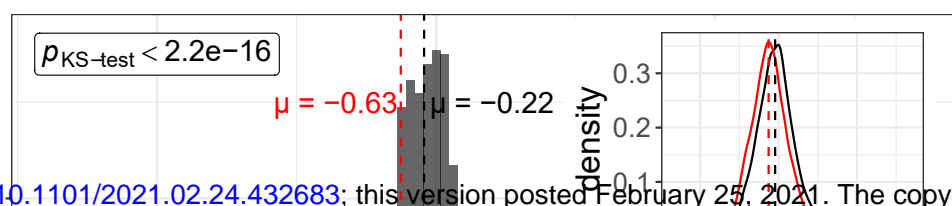

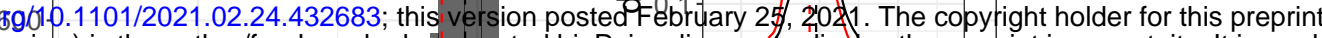
(which was not certified by peer review) is the author/funder, who has granted bioRxiv a license to display the preprint in perpetuity. It is made
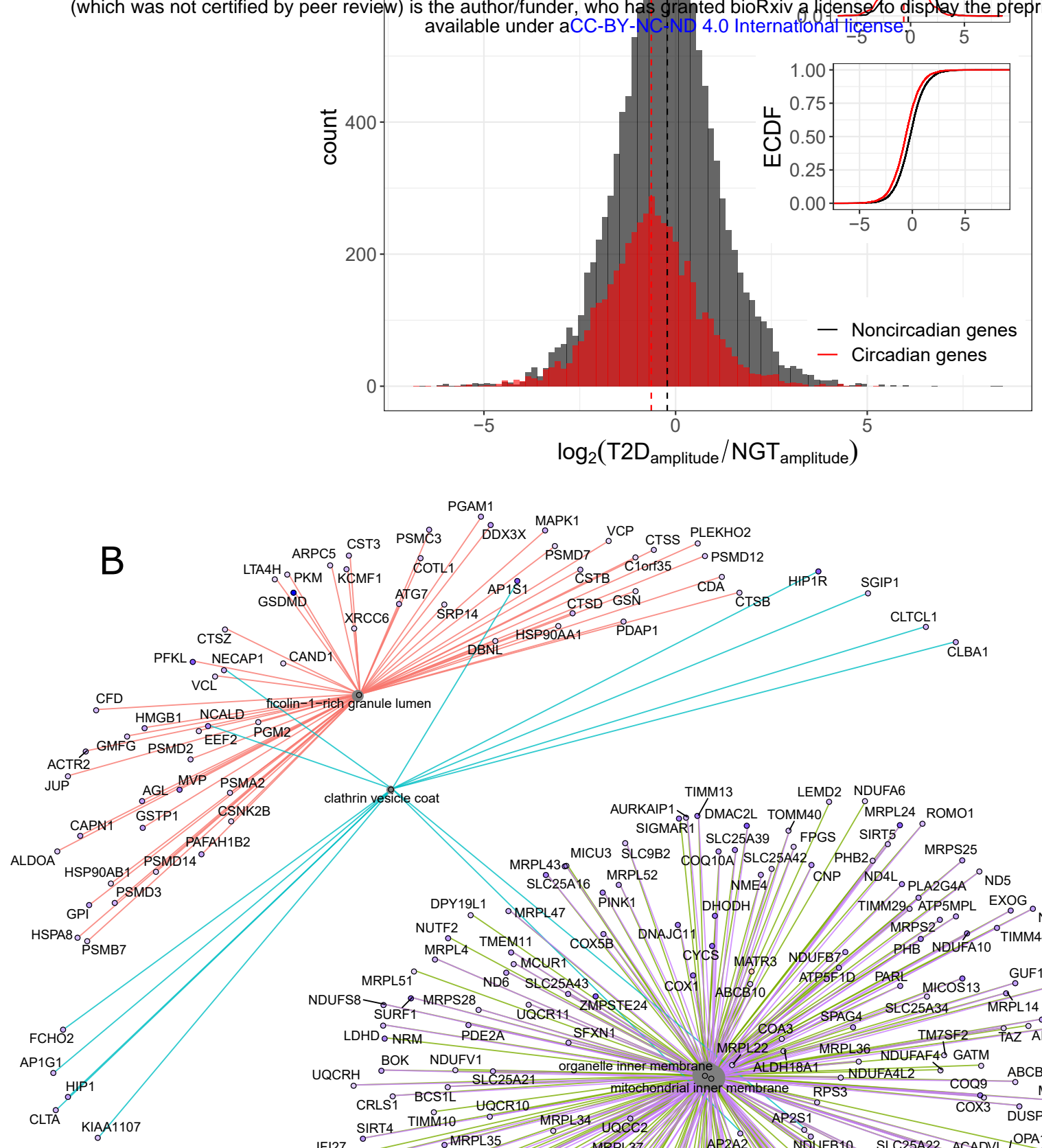

MRPL430 MICU3 SิLC9B2 COQ10A SLC25A42 PHB2D MRPS25

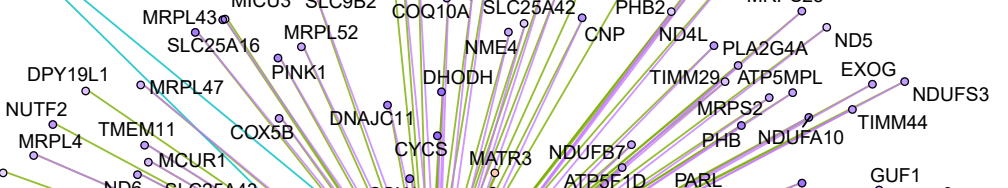

category

- clathrin vesicle coat

- ficolin-1-rich granule lumen

- mitochondrial inner membrane

- organelle inner membrane

size

- 14

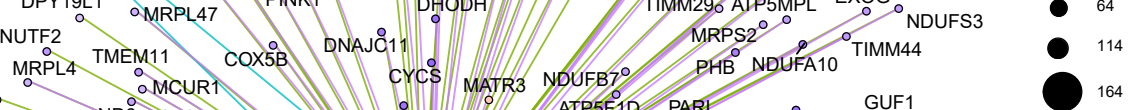

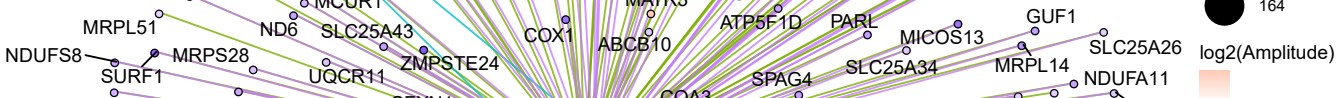

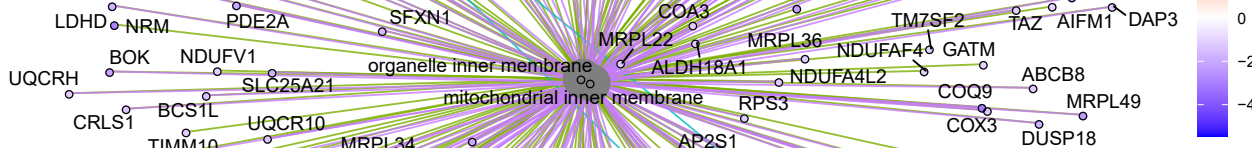

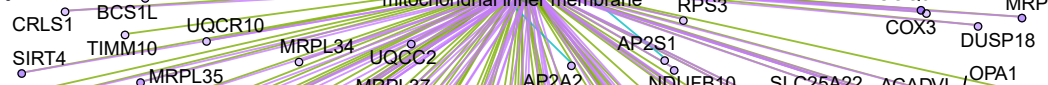

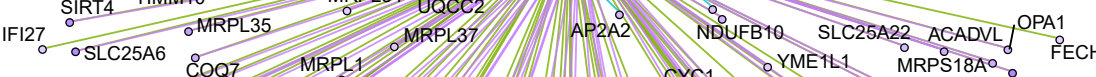

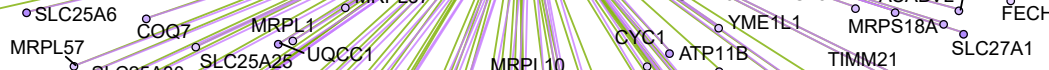

BDH1 10SLC25A30 SLC25A25 UQCET YQCRC1 MRPL10 COX5A MRPL55 SUN10 THMM21 O ORPS31

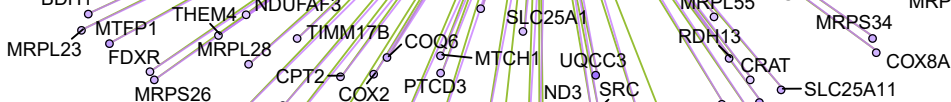

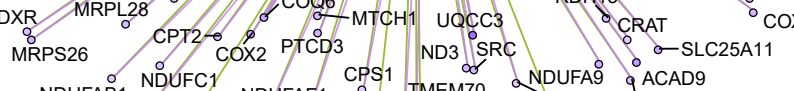

NDUFAB1 NDUFC1 NDUFAF1 CPS1 TMEM70 LNDUFA9 @ACAD9

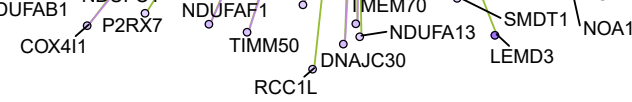

C

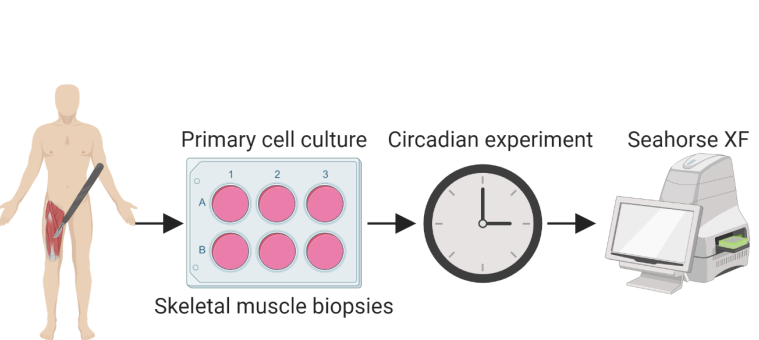

Type 2 Diabetic \& Normal glucose tolerant subjects
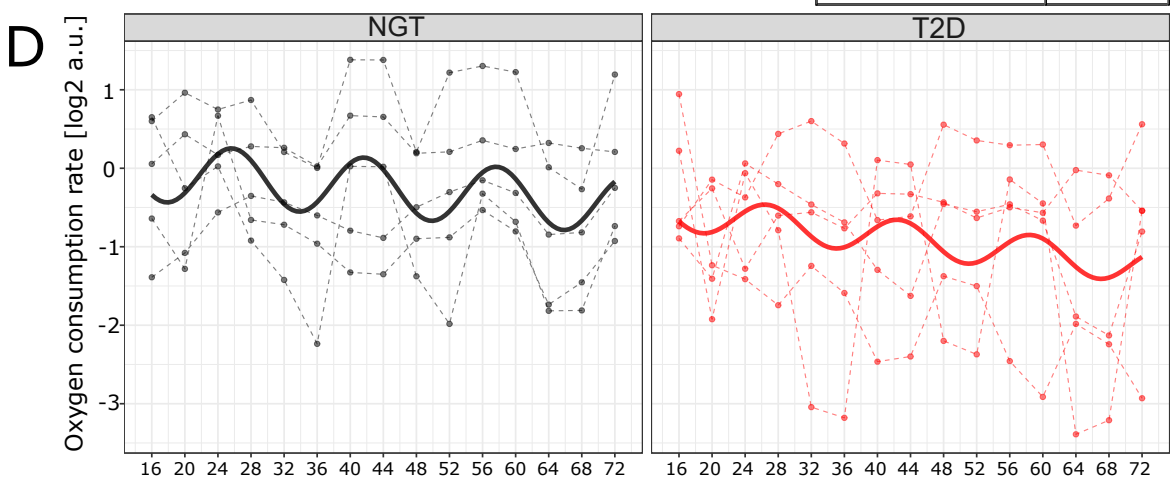

Time $[\mathrm{h}]$ 

organelle inner vesicle coat

Skeletal muscle biopsies

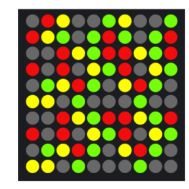

Microarray-mRNA

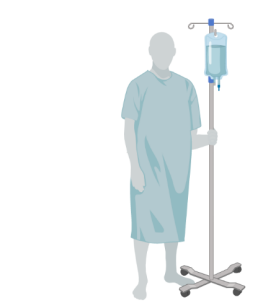

Hyperinsulinaemic-euglycaemic clamp

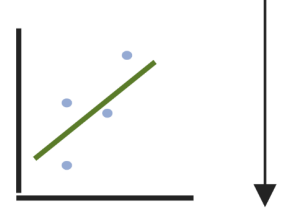

Correlation M-value

B

Insulin sensitivity

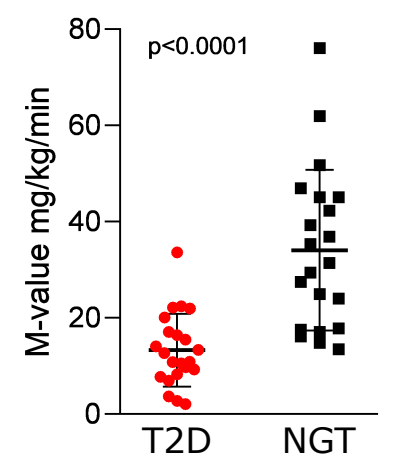

D mitochondral membrane part aCC-BY-NC-ND 4.0 International license. respiratory chain
mitochondrial protein complex

mitochondrial protein complex
al membrane protein complex

respiratory chain complex

DNA replication factor A complex replication fork-
nuclear replication fork-<smiles>[PbH]</smiles>

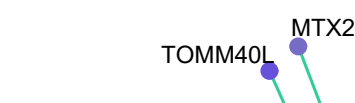

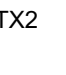

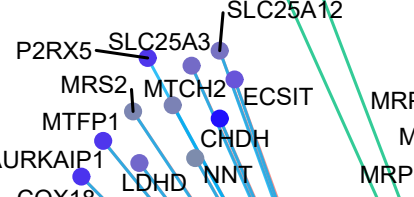
GOQ3 ${ }_{\text {GUF1 }} \$$ PD1 1 GRPEL1

SUCLG1 NUDT9 ACAD8 ALKBH7 /NARS2

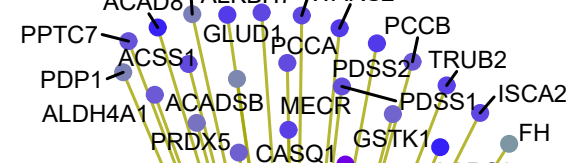

MDH2 CASQ QYYMMT LARS2 OACAD10 MTRF1L LYRM7 ALDHTA1 DNAJA3 VDAC2 PHX30 10 GL GLRX5 No NAXE FASTKO GLRX2 VIU TUF GCOH ALDHGAT CHPF PL47 MRPS9 CLPX CS IFAM ADHFE1 PPA2 MRPL17MMRP S33 MIRPL 15 ACOF11,POLDIP2 ACO2 MRPS6 MRP 16 MRPL41 mitochondrial matrix LIAS DECR1 MMAB CYCS MRPL21. MRPL 36 MRPL 19 MRPL35 AMRPL4 L L MRPS5 PPIE LMIPEP HIBADH

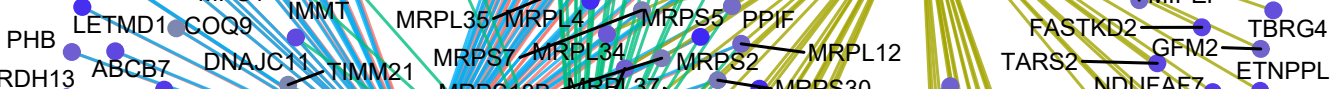

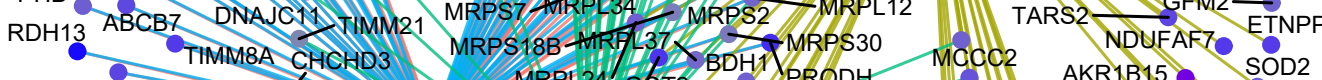
UQCC1 $20 \times 7 \mathrm{~B}$ ofganelle inner membrame 24 GST2

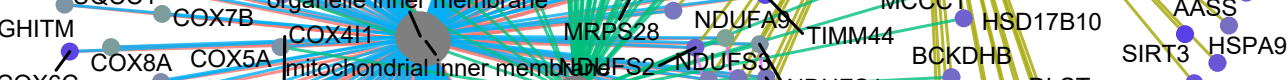

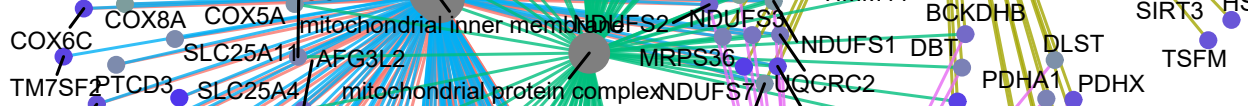

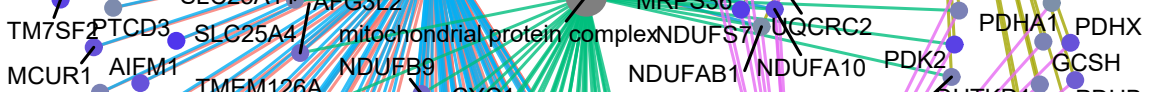

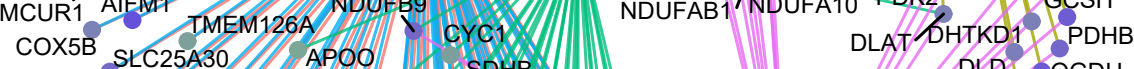

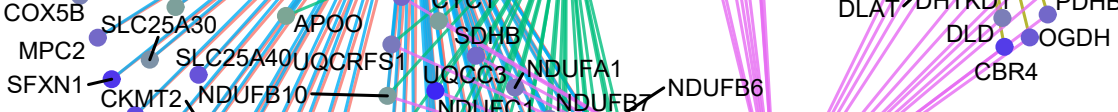

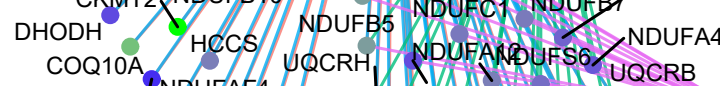
TAZ YNDUFAF4 SDHA NDUFA5 NDUFS4 SDHC oxidoreductase complex COX7A2\% SDHA UQCRC1

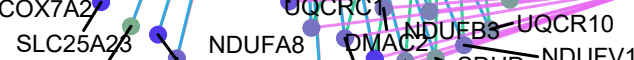
NDUFA8 PMAC2 COX7C NDUFV2 NDUFA6

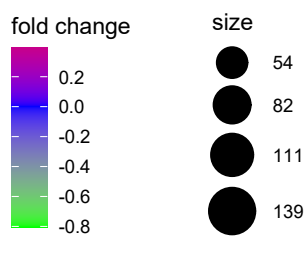
category

— mitochondrial inner membrane — mitochondrial matrix

— mitochondrial protein complex — organelle inner membrane — oxidoreductase complex

Figure 4 
A

ChIP-Seq 


\section{A}

NAD+/NADH?

Mitochondrial mémbran ROS?

D S Skeletal muscle clock

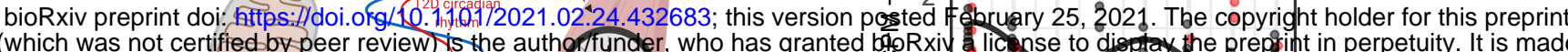

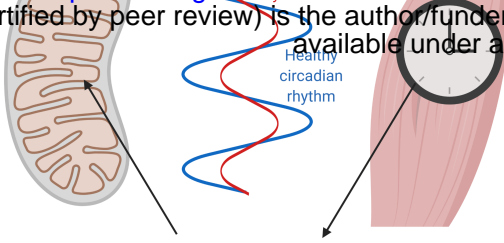

SODOC
B

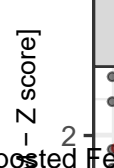

HIF1A

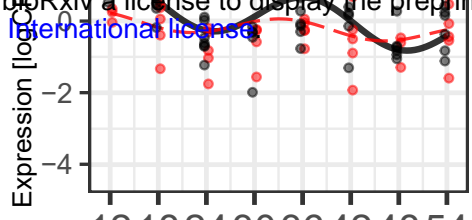

1218243036424854

Time [h]

Group:- $\rightarrow$ NGT $\rightarrow$-T2D Rhythmic:--NO -YES
C
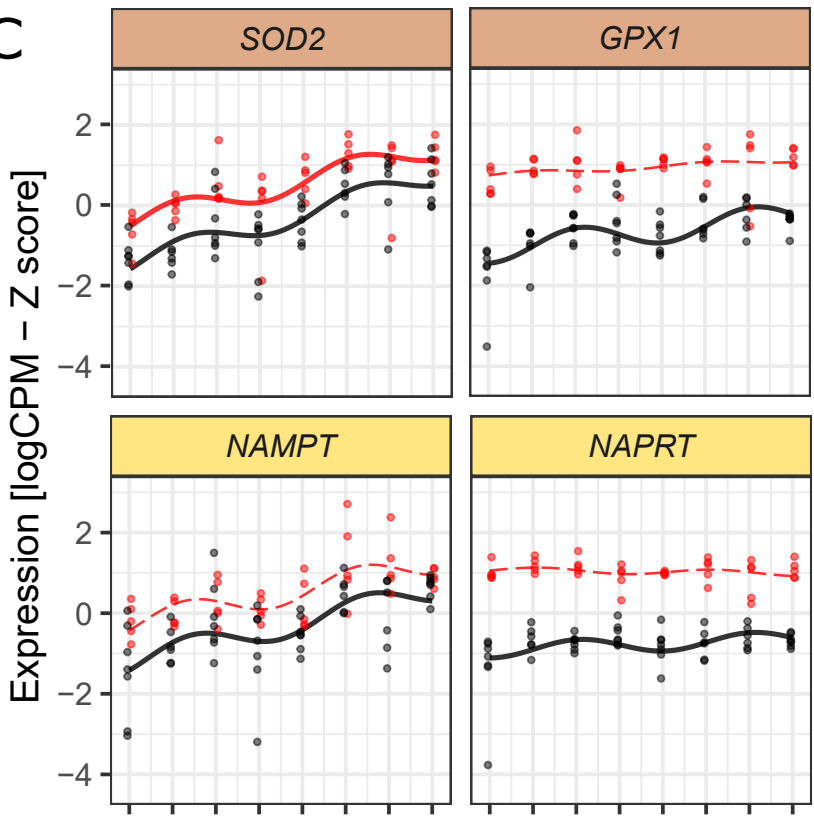

$1218243036424854 \quad 1218243036424854$
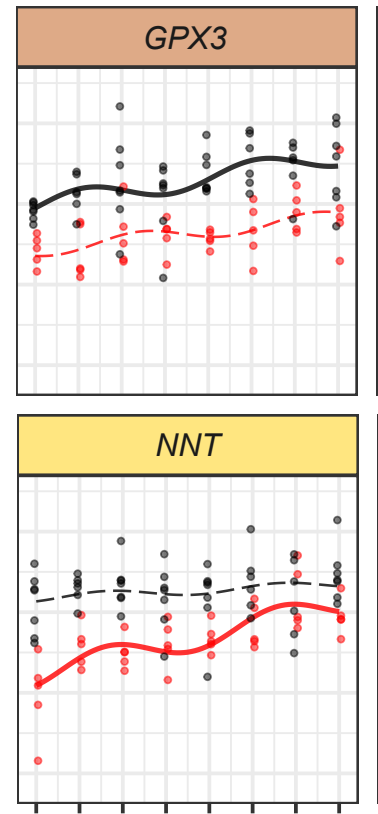

1218243036424854 Time [h]

$$
\text { group } \rightarrow \text { NGT } \rightarrow \text { T2D Rhythmic }- \text { - NO - YES } \square \text { Anti-ROS }
$$
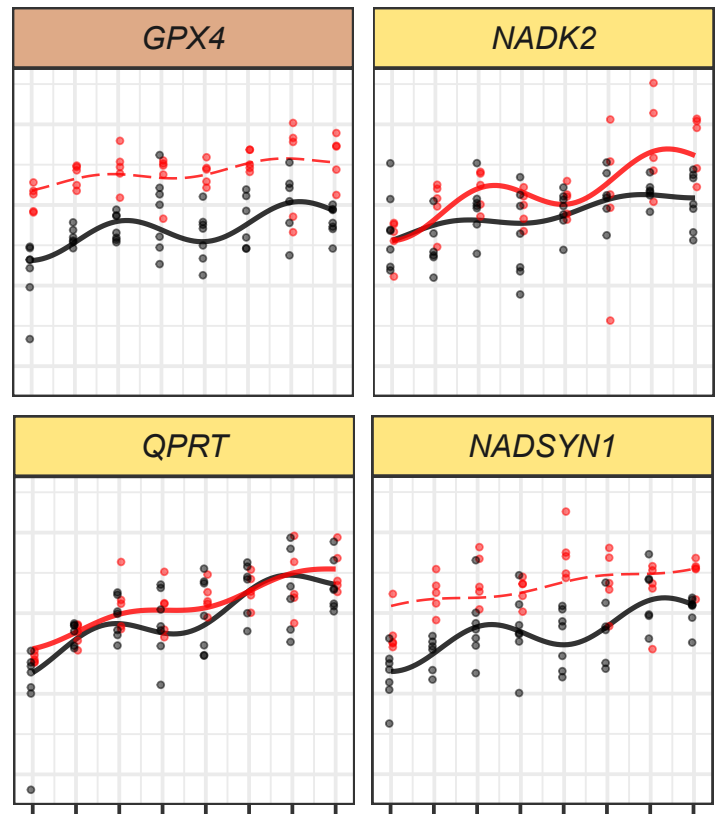

1218243036424854

$\square \mathrm{NAD}+/ \mathrm{NADH}$

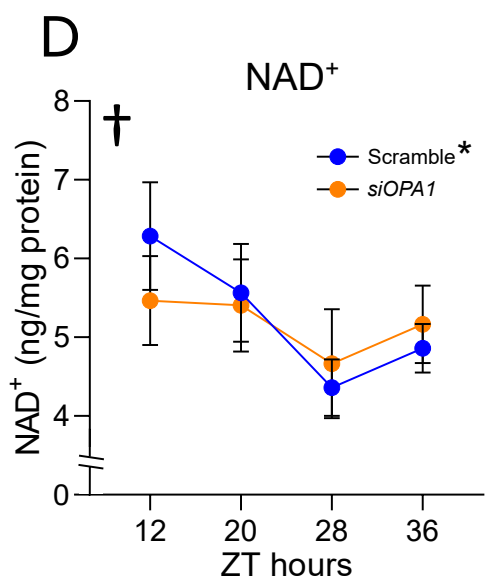

$\mathrm{E}$
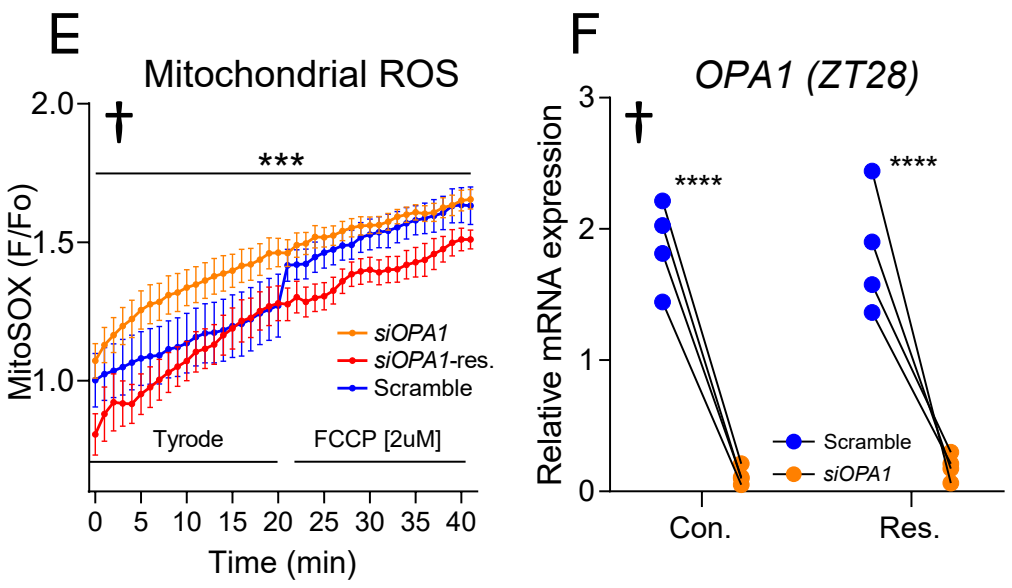

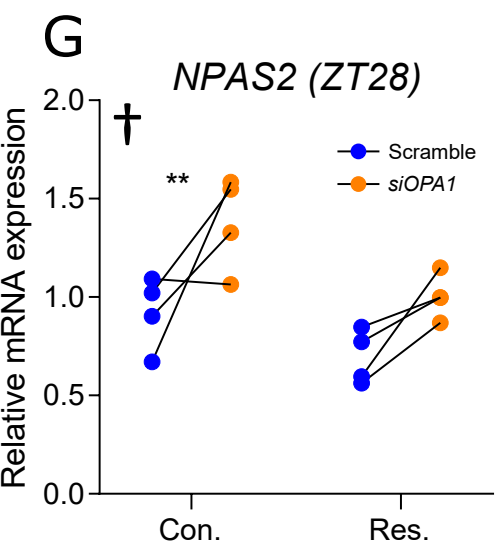




\section{A. Participant}

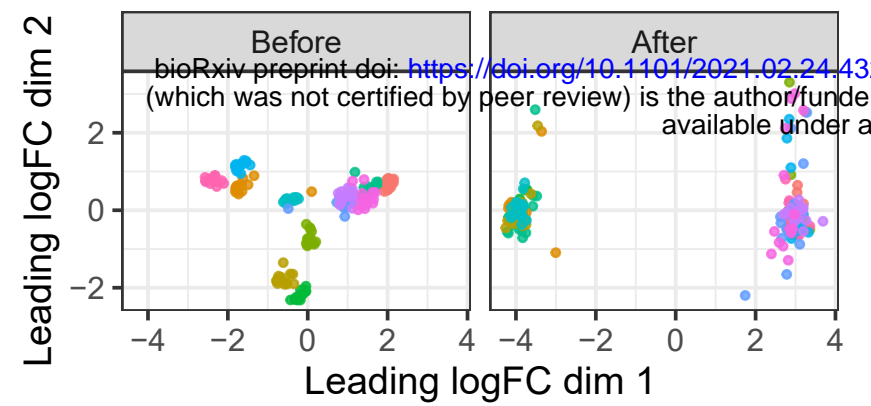

$m$

After

Participant

\section{B. Disease}
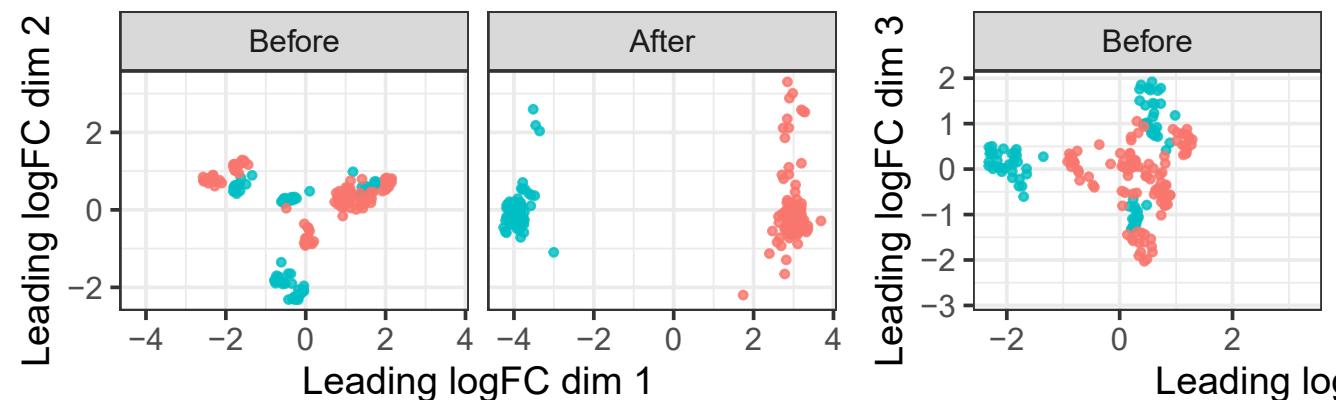

\section{Treatment}

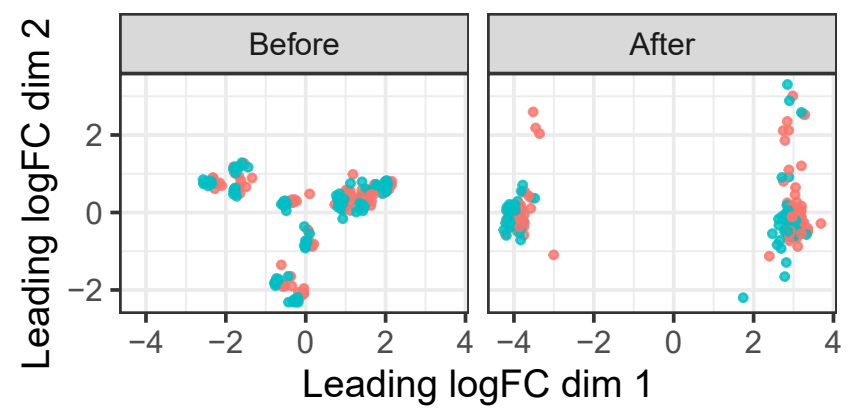

D. Time
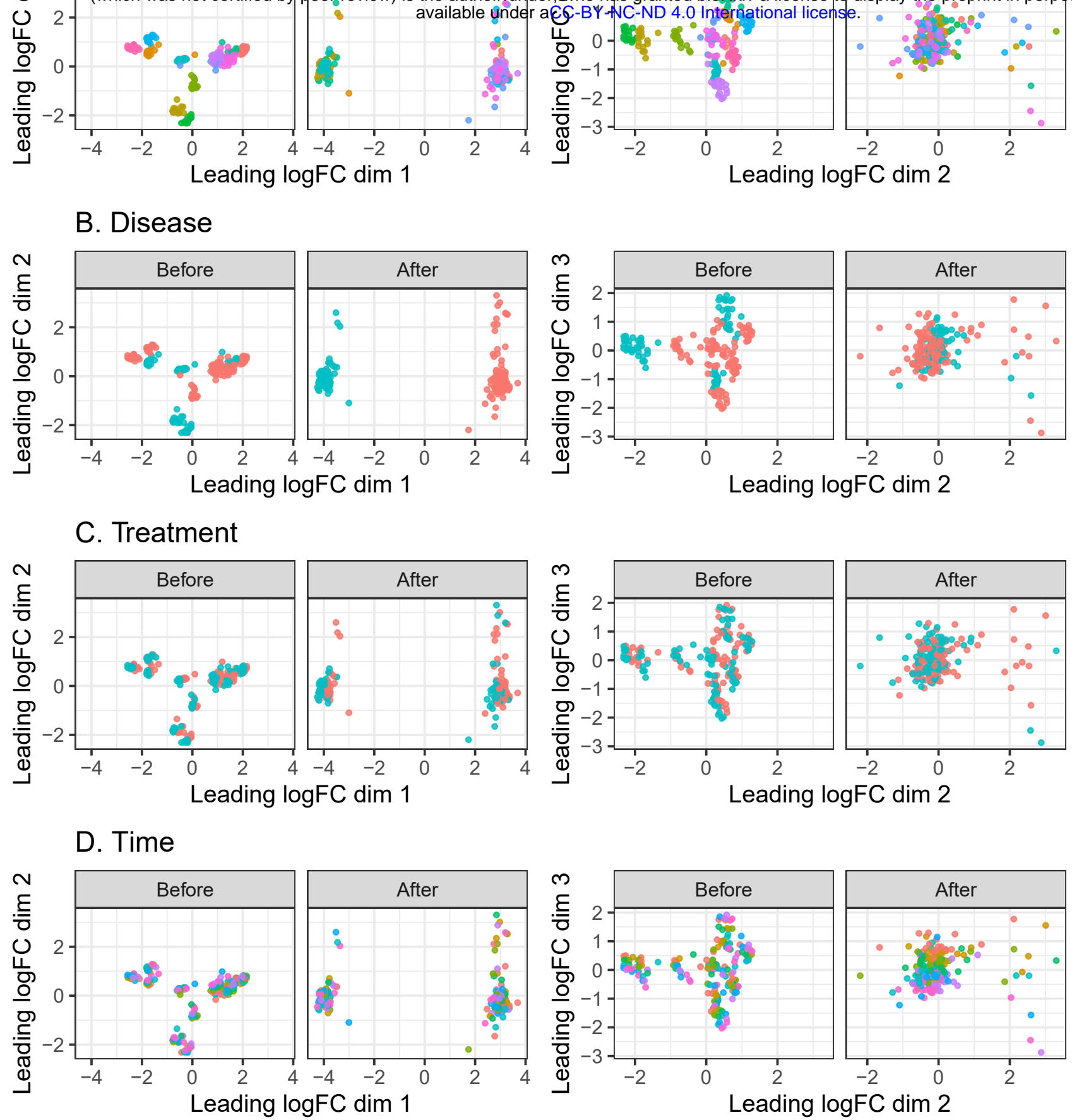

Disease

- NGT

- $\mathrm{T} 2 \mathrm{D}$

Treatment

- Control

- $H G$

Time

- 12

- 18

- 24

- 30

- 36

- 42

- 48

54

\section{Supplementary Figure 1}




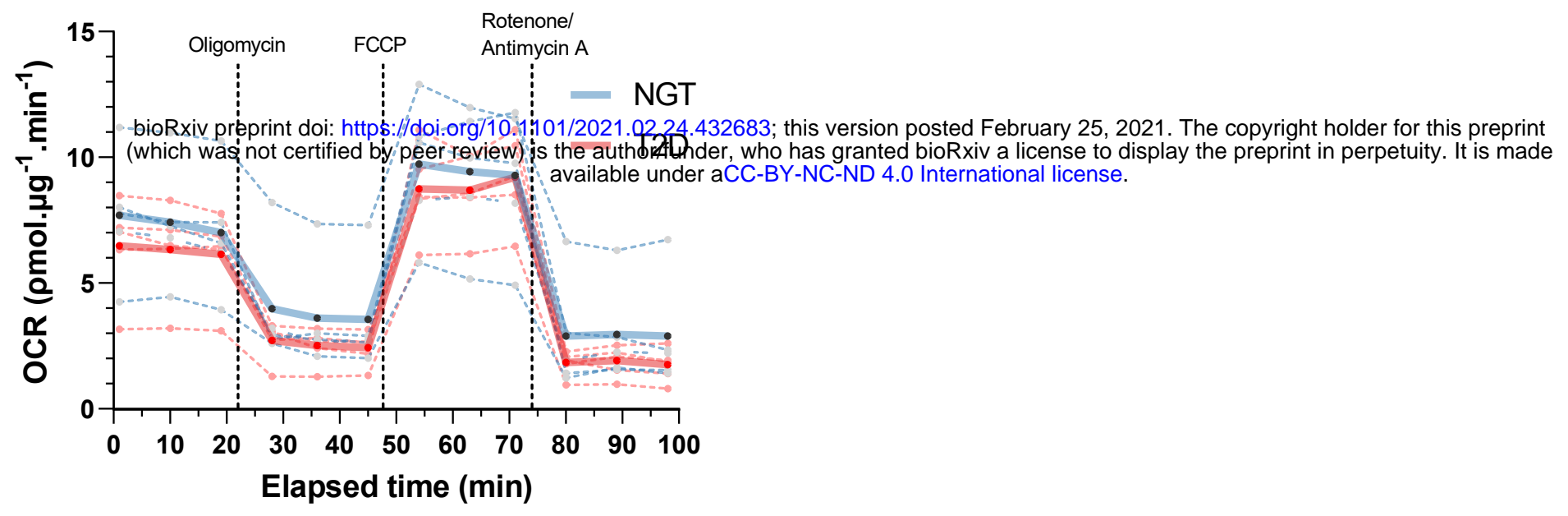

Supplemental figure 2 


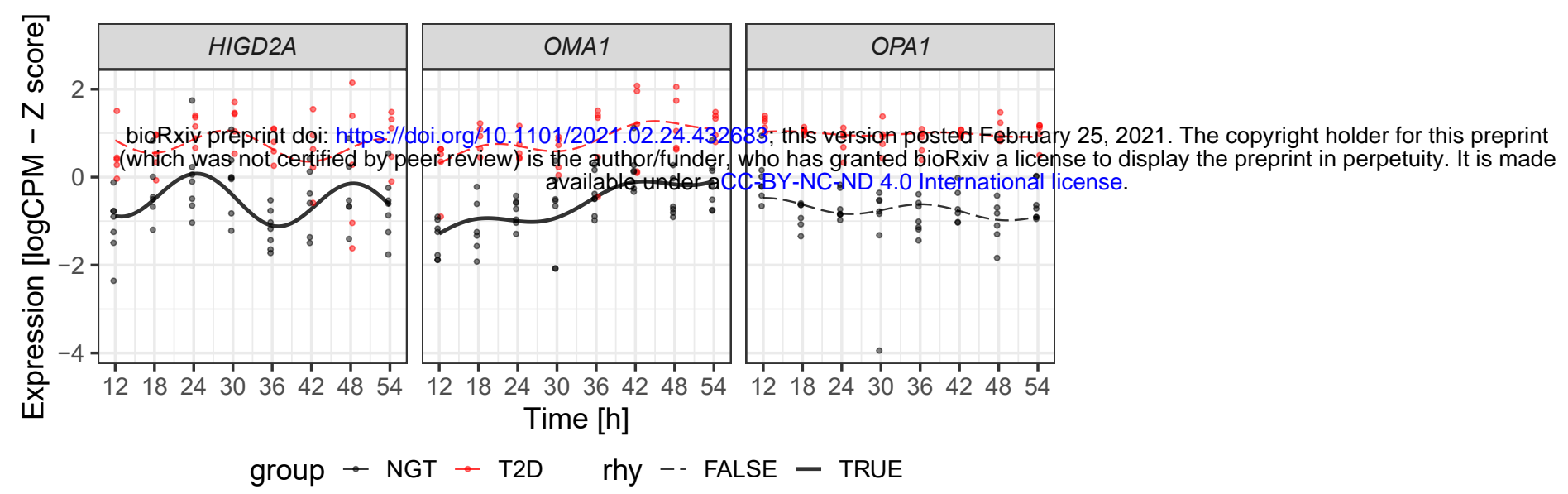

\section{Supplemental figure 3}

